\title{
A Novel CRISPR-Engineered, Stem Cell-Derived Cellular Vaccine
}

2

3 Krishnendu Chakraborty ${ }^{\mathrm{b}}$, Abishek Chandrashekar ${ }^{\mathrm{c}}$, Adam Sidaway ${ }^{\mathrm{b}}$, Elizabeth

4 Latta $^{\mathrm{b}}$, Jingyou Yuc, Katherine McMahanc, Victoria Giffinc, Cordelia Manickam,

5 Kyle Krollc, Matthew Mosher ${ }^{c}$, R. Keith Reeves ${ }^{c}$, Rihab Gam $^{b}$, Elisa Arthofer ${ }^{b}$,

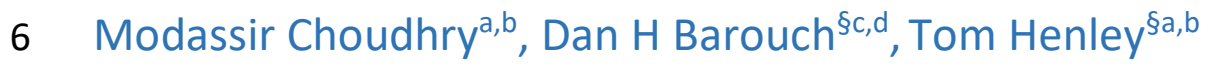

7

8 apraesidium Bioscience, Inc. New York, NY, USA

9 bIntima Bioscience, Inc. New York, NY, USA

$10{ }^{\mathrm{c} C e n t e r}$ for Virology and Vaccine Research, Beth Israel Deaconess Medical Center, Harvard Medical School, Boston, MA, USA

${ }^{d}$ Ragon Institute of Massachusetts General Hospital, Massachusetts Institute of Technology, and Harvard University, Cambridge, MA, USA

14

15

16

17

18

19

20

21

22

23

24

25

26

27

28

29

30

31 
COVID-19 has forced rapid clinical translation of novel vaccine technologies, principally mRNA vaccines, that have resulted in meaningful efficacy and adequate safety in response to the global pandemic. Notwithstanding this success, there remains an opportunity for innovation in vaccine technology to address current limitations and meet the challenges of inevitable future pandemics. We describe a universal vaccine cell (UVC) rationally designed to mimic the natural physiologic immunity induced post viral infection of host cells. Induced pluripotent stem cells were CRISPR engineered to delete MHC-I expression and simultaneously overexpress a NK Ligand adjuvant to increase rapid cellular apoptosis which was hypothesized to enhance viral antigen presentation in the resulting immune microenvironment leading to a protective immune response. Cells were further engineered to express the parental variant WA1/2020 SARS-CoV-2 spike protein as a representative viral antigen prior to irradiation and cryopreservation. The cellular vaccine was then used to immunize non-human primates in a standard 2-dose, IM injected prime + boost vaccination with $1 \mathrm{e} 8$ cells per $1 \mathrm{ml}$ dose resulting in robust neutralizing antibody responses (1e3 nAb titers) with decreasing levels at 6 months duration. Similar titers generated in this established NHP model have translated into protective human neutralizing antibody levels in SARS-Cov-2 vaccinated individuals. Animals vaccinated with WA1/2020 spike antigens were subsequently challenged with $1.0 \times 10^{5} \mathrm{TCID}_{50}$ infectious Delta (B.1.617.2) SARS-CoV-2 in a heterologous challenge which resulted in an approximately 3-log order decrease in viral RNA load in the lungs. These heterologous viral challenge results reflect the ongoing real-world experience of original variant WA1/2020 spike antigen vaccinated populations exposed to rapidly emerging variants like Delta and now Omicron. This cellular vaccine is designed to be a rapidly scalable cell line with a modular poly-antigenic payload to allow for practical, large-scale clinical manufacturing and use in an evolving viral variant environment. Human clinical translation of the UVC is being actively explored for this and potential future pandemics.

Key words: COVID-19; SARS-CoV-2; CRISPR; Stem Cell, iPSC, Vaccine, Virology; Vaccinology; ImmunoVaccinology; Universal Cell.

61 


\section{INTRODUCTION}

The COVID-19 pandemic has demonstrated the urgent need for new innovations in vaccinology to enable the rapid development of novel vaccines against emerging viral variants that engender robust and long-lasting immune protection. The unprecedented success of both mRNA and adenoviral vaccines have established the capability of a rapid global vaccination program ${ }^{1,2,3}$. However, the waning antibody responses seen with these emergency-use authorized vaccine technologies, and the need for vaccine boosters, has highlighted the requirement for further improvements in vaccine approaches to drive higher, longer-lasting protective immunity $4,5,6,7,8,9$. The newly emerging viral variants of SARS-CoV-2, and the evident reduced efficacy of the existing vaccines to protect against transmissible and symptomatic infection of these variants, also highlights the need for vaccines that can ideally deliver multiple variant antigens (polyvalency) and be rapidly manufactured at scale as soon as new viral variants are discovered ${ }^{10,11,12,13}$.

Theoretically, an ideal vaccine technology would have four core attributes, namely: hyperimmunity, self-adjuvancy, polyvalency and scalability. The first hyper-immunity is self-evident and speaks to the requirement of generating a robust humoral neutralizing antibody, and ideally a subsequent $\mathrm{T}$ cell amnestic response, such that protection remains durable. Self-adjuvancy, or conversely the absence of the need for exogenous excipients to elicit a hyper-immune response may prove to be a meaningful innovation in that the immune side-effects of current vaccines may be mediated by the non-target antigen specific adjuncts ${ }^{14}$. Thirdly, polyvalency or the ability to protect against multiple immunodominant epitopes, is a core feature of overlapping and orthogonal mechanisms of protection and is a core principle or antibiotic and antiviral therapy in infection to suppress underlying pathogenic genetic drift and mutation and acquired resistance ${ }^{15,}{ }^{16}$. Lastly, scalability or the ability to deliver preventative doses of vaccines in an economic, large scale and clinically relevant fashion in both the developed and developing worlds, is a sine qua non of any human vaccine.

Current mRNA, protein, and viral vector-based vaccines have certain limitations, such as their requirement for excipient adjuvants to activate the recipient immune system, or to deliver the viral antigenic payload ${ }^{17,18}$. These include the artificial lipid nanoparticles delivering the mRNA, or MF59, AS03, Alum, ISCOMATRIX, and Matrix-M chemical emulsions for example, or the adenoviral protein antigens themselves that stimulate innate immune cell activation ${ }^{18,19,20,21,22,23,24}$. Adjuvants are required to increase the effectiveness of vaccines and their use can cause side-effects including local reactions (redness, swelling, and pain at the injection site) and systemic reactions (fever, chills, and 
with Thrombosis with Thrombocytopenia Syndrome (TTS) and Myocarditis, secondary to the current COVID-19 vaccines $25,26,27$.

The size constraint of the adenoviral vector genome, and the limited length of stable mRNA that can be produced and packaged into nanoparticles, restricts the number and size of nucleic acidencoded antigens and epitopes that can be delivered in these vaccines ${ }^{28}$. Thus, these vaccines are constrained in their ability to provide multiple immunodominant proteins to address emerging pandemic variants, or to easily combine multiple pathogens into one vaccine.

To address some of the current limitations of vaccine technologies, we have developed a novel vaccine platform based on a CRISPR genetically engineered human stem cell, termed the Universal Vaccine Cell (UVC). The principal feature of this vaccine platform is to attempt to reproduce physiologic immunity that is engendered naturally through lytic viral infection and the resulting apoptosis of primary human cells. The platform is designed to deliver an almost unlimited antigenic payload within the context of a physiological apoptotic environment, to both release abundant antigen and simultaneously stimulate the host immune response. Here, we use the SARS-CoV-2 virus as a rigorous and timely test platform, to demonstrate that this self-adjuvanting, polyvalent UVC, can generate a robust and antigen-specific humoral immune response in vaccinated macaques. This hyper-immunogenic vaccine resulted in reduced viral loads in animals challenged with heterologous SARS-CoV-2 variant, which recapitulates the current experience of a population vaccinated against the initial parental WA1/2020 variant, yet now exposed to novel variants like Delta and Omicron ${ }^{29,30,31}$. 


\section{RESULTS}

Genetic engineering of iPS cells to create a cellular vaccine to deliver the SARS-CoV-2 spike antigen

To create a cellular vaccine platform to deliver abundant viral antigens and simultaneously engage host innate immune cells to present these antigens to lymphocytes, we attempted to create a cell with a hyper-immunogenic phenotype. We selected human iPS cells as the UVC cell line due to their stable genetics, non-transformed phenotype, ease of genetic engineering and capacity for rapid scalable propagation ${ }^{32,33}$. IPS cells also retained the unique ability for programmable differentiation into any cell lineage, thus retaining the future opportunity to explore differentiation of the UVC into different cell types that may have enhanced immunogenic properties ${ }^{34}$.

We first genetically engineered iPS cells to create an immunogenic phenotype by stable integration of the SARS-CoV-2 full length spike antigen into the AAVS1 safe-harbor locus using CRISPR/Cas9 gene editing (Fig. 1a). We selected the original and well-characterized WA1/2020 variant of SARS-CoV-2 and spike antigen sequence with mutation of the furin cleavage site and prolinestabilizing mutations that is identical to that in the current emergency-use authorized vaccines being deployed globally to vaccinate against COVID-1935,36,37 (Supplementary Fig. S1). By including the spike transmembrane domain sequence in the gene encoding this antigen, we were able to detect high levels of the viral spike on the cell surface of the engineered iPS cells (Fig. 1b). Spike protein was also readily observed in engineered cell lysates when measured by western blotting (Fig. 1c). The yield of antigen released upon lysis was quantified using a spike-specific ELISA assay and we observed an abundant and dose-dependent release of protein from the cells, which would equate to approximately $\sim 20$ micrograms of spike antigen protein delivered in a $1 \times 10^{8}$ cell vaccine dose of UVC (Fig. 1d).

To ensure robust delivery of this immunodominant antigen to the recipient immune system, we incorporate an apoptosis-inducing lethal irradiation step during vaccine manufacture by exposing the UVC cells to a $10 \mathrm{~Gy}$ dose of gamma radiation prior to cryopreservation and vaccination. Thus, when subjects are immunized with the UVC, we reasoned that the cells would undergo apoptosis and release the SARS-CoV-2 spike antigen into the immune microenvironment via production of apoptotic bodies (Fig. 1a). In theory, these apoptotic bodies will be phagocytosed by innate immune cells and antigen-presenting cells and presented to $\mathrm{T}$ and $\mathrm{B}$ lymphocytes to generate a spike antigen-specific immune response.

In addition to creating a mechanism for delivery of immunogenic antigens via apoptotic bodies, the irradiation of the UVC can be considered a safety feature as it renders the cells unable to 
proliferation or persist in vivo upon vaccination. In support of this, we observed a robust elevation in the proportion of apoptotic cells after 24 and 72 hours of culture of irradiated UVC, both using apoptotic dyes and flow cytometry (Fig. 1e) and by observation of cell morphology under the microscope (Fig. 1f). Furthermore, unlike non-irradiated UVC, irradiation prevented any detectible proliferation of the cells over 72-hours in culture as measured by proliferation dyes using flow cytometry (Fig. 1g).

\section{Incorporation of NK cell activation signals by genetic engineering to create a self-adjuvanting} vaccine cell

In addition to the proposed immunogenicity expected from apoptosis and release of immunogenic antigens upon vaccination, we attempted to increase the immunogenic potential further and incorporate a self-adjuvanting phenotype to the UVC. As a form of physiological cell death, apoptosis is generally non-inflammatory ${ }^{38}$. Therefore, to promote effective local inflammation and engage the innate immune system that can mobilize effector cells, we engineered the UVC to mimic a virally infected cell to be recognized and rapidly lysed by host innate immune cells, principally NK cells ${ }^{39,40}$. Many viruses attempt to evade immune recognition by limiting MHC-I cell surface expression to reduce the presentation of viral antigens to $C D 8^{+} T$ cells $s^{41,42}$. This "missing-self" signal can aid in the activation of NK calls and promote cytolysis, and therefore the iPS cells were engineered to completely remove MHC-I molecules from the cell surface via CRISPR knockout of the $\beta 2$ microglobulin (B2M) gene, a critical component of MHC class I molecules (Fig. 1h).

In vivo, lack of MHC-I on the target cell is not sufficient to trigger full NK cell activation alone and a further hallmark of cells undergoing stress or viral infection, is the expression of NK cell activating natural killer group 2 member D (NKG2D) ligands on their cell surface ${ }^{43}, 44$. Therefore, we further engineered the UVC using CRISPR to integrate a gene expression cassette in a safe-harbor locus to drive constitutive expression of the human MICA gene (MHC class I polypeptide-related sequence A), a potent activator of NK cells. Using flow cytometry, abundant levels of MICA could be detected on the surface of the engineered UVC (Fig. 1h).

\section{Rapid growth kinetics of engineered UVC}

Prior to irradiation and cryopreservation of the UVC ready for immunization, we evaluated the growth kinetics of the cells to confirm the capacity for rapid, scalable proliferation that would be needed for a vaccine technology to address the needs of a pandemic. IPS cells are known to have a relatively short 
202

203

204

205

206

207

208

209

210

211

212

213

214

215

216

217

218

219

220

221

222

223

224

225

226

227

228

229

230

231

232

233

234

doubling times in the range of $18-20$ hours $^{45,46}$, and we observed similar kinetics with an average exponential growth of $>50$-fold over a 7-day culture period (Fig. 1i). Thus, from a starting UVC number of $1 \times 10^{6}$ cells, the vaccine can be theoretically expanded to provide millions of doses in under 8 weeks, and even quicker if adapted to bioreactor manufacturing.

The consistent rapid cell growth of the UVC and the morphological similarity to unmodified iPS cells, suggested the UVC reattained broad characteristic of the iPS cells from which they are derived. We thus assessed the stem cell characteristics of the UVC after genetic engineering and rapid expansion to confirm that the cells have retained their original stemness-gene expression signatures without acquiring any detectible or obvious changes in phenotype beyond those introduced by genetic engineering. The expanded UVC expressing the SARS-CoV-2 spike antigen, human MICA ligand and CRISPR knockout of B2M, showed a similar level of expression of three important pluripotent transcription factors, NANOG, OCT4 and SOX2, suggesting they have retained a stem-cell like transcriptional profile (Fig. 1j). Engineered UVC also showed similar expression to control iPS cells for genes (DNC, Vimentin, HES5 and GATA6) that are known to increase in expression as iPS cells differentiate into mesoderm, endoderm and ectoderm liniage, confirming the UVC have a consistent undifferentiated, iPS cell gene expression profile, morphology, and growth characteristics ${ }^{47,48}$

Taken together, these data demonstrate that the CRISPR engineered UVC has the capacity to deliver abundant, full-length spike protein antigen in the context of an irradiated, apoptotic cellular vehicle. Via the genetically engineered absence of MHC-I and overexpression of MICA, the UVC has the potential for NK cell activation upon immunization, which may remove the need for excipient adjuvants to promote inflammation and immunogenicity. We first tested this hypothesis in vitro to measure the activation of NK cells by the UVC and the NK-mediated UVC cytolysis.

\section{Human and primate NK cell cytolysis of universal vaccine cells}

To further explore the impact of MHC-I loss and overexpression of NK cell ligands on recognition and killing of the UVC by NK cells, we performed a series of in vitro NK cell activation and cytolysis assays. When MHC-I was removed via B2M knockout alone, the UVC were robustly killed by human NK cells, which increased in an E:T ratio-dependent manner (Fig. 2a). We compared the level of UVC cytolysis to that observed with the MHC-deficient K562 leukemia cell line, known to be potent targets for NK cell killing, and found a similar level of cytolysis confirming the MHC-I deficient UVC are readily targeted by NK cells. We extended this analysis to macaque NK cells and found that while control iPS 
Immunogenicity of universal vaccine cells in vaccinated macaques by the NK cells (Fig. 2b). 2d).

cells (expressing MHC-I) show low levels of killing, the MHC-I Knockout UVC were lysed more readily

To assess the relative contribution of overexpressing NK activating ligands on UVC cytolysis by macaque NK cells, we performed an analysis of UVC cells transiently overexpressing different NKG2D ligands, including MICA, MICB and UL16 binding protein 1 (ULBP1). While the levels of macrophage

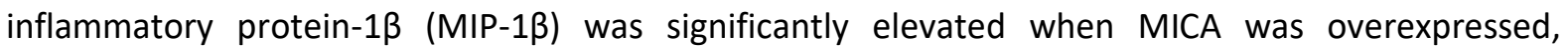
proinflammatory and activation markers for NK cells were generally the same regardless of ligand overexpression (Fig. 2c). With stable overexpression of MICA by CRISPR engineering, we confirmed a significant increase in total responding macaque NK cells and a significant elevation in MIP-1ß (Fig.

253

254 To evaluate the immunogenicity of the UVC and the vaccine's ability to engender a humoral immune response, we immunized cynomolgus macaques and followed the production of neutralizing and spike-specific antibodies over a 10-week period, and a duration follow up at 6 months. We immunized 9 macaques, aged 6-12 years old, with either $1 \times 10^{7}$ UVC $(n=3)$ or $1 \times 10^{8}$ UVC $(n=3)$ expressing the WA1/2020 SARS-COV-2 spike antigen, and sham controls $(n=3)$. Macaques received a prime dose immunization by the intramuscular route without adjuvant at week 0 , followed by a boost dose immunization (same cell number as prime dose) at week 6 (Fig. 3a). Neutralizing antibody responses were assessed using a pseudovirus neutralization assay 49, 50,51,52, and we observed neutralizing antibodies in all UVC vaccinated macaques at week 2 that further increased by week 4 (Fig. 3b). The higher dose of $1 \times 10^{8} \mathrm{UVC}$ resulted in the most robust titers of neutralizing antibodies at all timepoints tested. Following UVC boost dose immunization at week 6, neutralizing antibody titers elevated further, reaching close to $1 \times 10^{3}$ titers with the higher $1 \times 10^{8}$ cell dose. Six months after the initial UVC immunization, neutralizing antibody showed a durable response, and levels in macaques immunized with the $1 \times 10^{8}$ UVC dose remained elevated beyond that seen with the initial prime UVC dose. We also observed robust spike-specific and receptor-binding-domain (RBD)-specific antibody titers, as 
measured by enzyme-linked immunosorbent assay (ELISA) in vaccinated macaques (Fig. $3 \mathrm{c}$ and d). These antibody responses and durability at 6 months in the $1 \times 10^{8}$ dose, were like those seen with the neutralizing antibody titers, and thus collectively demonstrating that the UVC vaccine can engender a robust humoral response against the SARS-CoV-2 spike antigen with demonstrable durability. At 6 months after immunization, detectible levels of neutralizing antibodies against Beta and Delta were also observed, albeit lower than seen with the immunizing antigen variant WA1/2020 spike, suggesting humoral immunity is also generated against SARS-CoV-2 variants (Fig. 3e). This prompted us to assess the protective immunity of the humoral immune response generated by UVC immunization in the context of a SARS-CoV-2 heterologous infection challenge.

Humoral immune responses in vaccinated macaques after heterologous SARS-CoV-2 challenge

In a second non-human primate study, we immunized rhesus macaques, aged 6-12 years old, with the higher $1 \times 10^{8}$ dose of UVC ( $\left.n=6\right)$ expressing the SARS-CoV-2 WA1/2020 spike antigen, and sham controls $(n=6)$, and this time followed the production of neutralizing and spike-specific antibodies over an 8-week period (Fig. 4a). At week 8, the macaques were challenged with $1.0 \times 10^{5} 50 \%$ tissue culture infectious dose $\left(\mathrm{TCID}_{50}\right)$ of heterologous SARS-CoV-2 B.1.617.2 (Delta) by the intranasal and intratracheal routes ${ }^{51,52}$. Viral loads in bronchoalveolar lavage (BAL) and nasal swabs were assessed over 10-days by reverse transcription PCR (RT-PCR) specific for subgenomic mRNA (sgRNA), which is thought to measure replicating virus ${ }^{52,53}$. Sham controls showed a median peak of 5.39 (range 4.605.88) $\log _{10}[\operatorname{sgRNA}$ (copies per $\mathrm{ml}$ )] in BAL samples (Fig. $4 \mathrm{~b}$ and d). Partial protection was observed in macaques immunized with the UVC as a significantly lower level of virus was detected in BAL samples, with a median peak of 2.78 (range 1.70-4.63) $\log _{10}[\mathrm{sgRNA}$ (copies per $\mathrm{ml}$ )], representing a 2.81 log reduction in virus in UVC vaccinated animals. A significant reduction in virus (0.96 log reduction) was also observed in nasal swabs from UVC immunized macaques when compared to sham controls, albeit lower in magnitude than seen when comparing BAL samples (Fig. 4c and e).

While neutralizing antibody titers specific for the WA1/2020 variant spike was high in both macaque immunization studies (Fig. 3 and supplementary Fig. S2), the titers specific for other SARSCoV-2 variants (Beta and Delta) was lower, which is to be expected given the divergence in antigen protein sequence. Thus, the partial protection seen in animals immunized with WA1/2020 spike UVC and challenged with B.1.617.2 (Delta) SARS-CoV-2, is also expected given the heterologous nature of the challenge. We would predict a more robust and complete reduction in virus from animals immunized with UVC and challenged with SARS-CoV- 2 in which the antigen and variant are matched. 
Collectively these data demonstrate that a prime and boost dose of $1 \times 10^{8} \mathrm{WA} 1 / 2020$ Spike expressing UVC promote a robust antigen-specific antibody response with levels of neutralizing antibodies and durability similar to the current approved COVID-19 vaccines ${ }^{49,54,55}$, and this can lead to partial protective immunity in a heterologous WA1/2020 versus Delta SARS-CoV-2 virus challenge.

307

308

309

310

311

312

313

314

315

316

317

318

319

320

321

322

323

324

325

326

327

328

329

330

331

332

333

334

335 


\section{DISCUSSION}

339 The urgent global need for improved vaccine technologies to meet future pandemics is driving a 340 renaissance of innovation in vaccinology. COVID-19 has demonstrated the rapid pace at which viral 341 mutations can accumulate and new variants emerge that can escape the protective efficacy of existing 342 vaccines designed against earlier viral antigen sequences ${ }^{10,11,29,30,31,56,57}$. To address the need for 343 novel vaccine technologies, we have developed the first cellular vaccine, to generate hyper-immunity 344 via self-adjuvancy through apoptosis and NK cell-mediated cytolysis within the immune microenvironment.

Our data demonstrate that the UVC vaccine platform can induce robust neutralizing antibody responses in vaccinated macaques when delivering the SARS-CoV-2 WA1/2020 (original variant ${ }^{58}$ ) fulllength membrane-bound Spike protein, with mutation of the furin cleavage site and two prolinestabilizing mutations $\mathrm{s}^{35,36,37}$. The antibody titers and resulting immunogenicity were on par with the neutralizing antibody titers demonstrating immune protection with the emergency use authorized and now commercially available mRNA vaccines (1e3 nAb titer) ${ }^{49,54,55}$. We also show that the UVC delivering the WA1/2020 Spike antigen enhances neutralizing antibodies and (RBD)-specific binding antibodies specific for the B.1.617.2 (Delta) and B.1.351 (Beta) variants of SARS-CoV-2. This robust and specific humoral response can partially protect rhesus macaques vaccinated with a prime and boost dose of WA1/2020 Spike UVC in a heterologous variant challenge with infectious B.1.617.2 (Delta) SARS-CoV-2 and engender a more rapid clearance of viral RNA in the BAL. A limitation of the WA1/2020 UVC in a Delta heterologous challenge was the absence of any meaningful protection in nasal swabs. This discordance between vaccine variant, versus virus variant, is the principal challenge facing current vaccinated populations and might suggest the reason for ongoing infectious spread but more limited morbidity and mortality against these emerging variants ${ }^{29,}{ }^{31}$.

As regards duration of protection, the theoretical hyper-immunity postulated by creating a self-adjuvanting, hyper-immune UVC established robust initial nAb titers. When these animals were rechallenged 6-month later, the nAb response stayed robust at the higher, and now established for clinical use, 1e8 UVC dose. Moreover, the persistent nAb response at 6-months remained robust for SARS-CoV-2 WA1/2020, Beta and Delta variants.

As regards intrinsic safety, the UVC undergoes lethal irradiation during manufacture and rapid apoptosis in the immune microenvironment upon vaccination. This is the principal mechanism of efficacy of the UVC, and fortunately its most redeeming safety feature, by virtue of the impossibility of in vivo persistence and teratogenicity of the cellular antigen carrier. The irradiation-induced 
371 introduce cell surface expression of the NKG2D ligand MICA, making the UVC potent targets for host

372 NK cells. Recruited NK cells will likely recognize the UVC as virally infected cell through MHC-I absence

373 and MICA activation of NKG2D signaling to mediate a direct killing effect and release of protein

374 antigen ${ }^{59}$. The apoptosis and NK-mediated cytolysis enables the UVC to be a self-adjuvanting vaccine

375 vector, without the need for additional chemicals adjuvants or additional foreign antigens. Thus, the

376 UVC may mimic the physiological engagement of the immune system typical of virally infective cells

377 within the tissues of an individual suffering with the disease. The use of a living cellular antigen vehicle,

378 as opposed to a lipid nanoparticle or other such inanimate construct, can potentially recapitulate

379 natural immunity without the need for exogenous adjuvants, which may portend greater safety

380 against autoimmune complications. Theoretically, and unproven and unprovable at this time absent

381 human clinical data, the natural physiologic nature may lead to an improved safety profile for the UVC.

The CRISPR genetic engineering to render the UVC highly immunogenic and self-adjuvanting,

383

384

385

386

387

388

389

390

391

392

393

394

395

396

397

398

399

400

401

402

403

404 also presents a unique opportunity to address antigen polyvalency. Unlike mRNA or DNA vaccines, or recombinant, replication-incompetent viral vector vaccines, that have a size limit of encoded antigen or the number of independent antigens they can deliver, the UVC can be engineered to express and deliver a much higher number of full-length protein antigens. Thus, there is the ability to create polyvalency against multiple epitopes in a rapid modular gene cassette fashion through CRISPR engineering of the iPS cell genome.

A perceived limitation of the UVC technology may be the seemingly complex and costly nature of developing and manufacturing a live human cell as a vector for vaccination at scale. However, the UVC is a cell line, not a complex cell therapy, and can thus be scaled within appropriate parameters for such a biologic agent. Once pathogen antigens have be genetically engineered, the UVC cell line can be expanded rapidly to scale with predictable growth kinetics and QA/QC controls. The modular nature of the UVC and the ability to integrate emerging viral antigens into the cellular genome using CRISPR, can allow scalable manufacture of new polyvalent vaccines to address emerging variants. In fact, the genetic engineering of the UVC cells can be accomplished in a matter of weeks prior to exponential cell culture expansion to create millions of clinical doses. As a test of the rapid and modular manufacturing of the platform, at the time of this publication, the authors have begun engineering a polyvalent UVC against the SARS-CoV-2 Omicron variant (B.1.1.529) (Fig. 5b).

A limitation of our study is that we have yet to observe the generation of a robust $T$ cell response in vaccinated animals. To put this into broader clinical context, the measurable albeit modest CD8-specific $T$ cell responses seen with the adenoviral and mRNA vaccines have not resulted in $n A b$ titers and duration of protection longer than 6-9 months ${ }^{8,60}$. One potential hypothesis to begin to establish a clinically meaningful amnestic T cell response is to explore novel non-spike antigens, thus 
405

406

407

408

409

410

411

412

413

414

415

416

417

418

419

420

421

422

423

424

425

426

427

428

429

430

431

432

433

434

435

436

437

leveraging the simultaneous polyvalency of the UVC platform. The UVC allows vaccination with multiple SARS-CoV-2 antigens, including immunodominant T cell epitopes such as those in the nucleocapsid and viral accessory proteins ${ }^{61,62,63}$. In the context of COVID-19, additional studies will evaluate the capability of UVCs delivering polyvalent T cell epitopes such as the Nucleocapsid, ORF and Membrane proteins, to generate a CD8 cytolytic and CD4 Helper T cell response in vaccinated macaques to engender duration of protection via T cell amnestic response. Beyond COVID-19, the potential of the theoretically unlimited antigenic payload of a cellular vaccine would allow for "polypathogenic polyvalency" and the creation of a single seasonal respiratory vaccine, which could include influenza, RSV, and pan-coronavirus viral antigens ${ }^{64}$.

In summary, these data establish the first cellular vaccine platform and demonstrate that immunization with a WA1/2020 SARS-CoV-2 spike expressing UVC vaccine elicits robust neutralizing antibody titers and provides partial protection against heterologous Delta SARS-CoV-2 challenge in rhesus macaques. Establishing this novel cellular vaccine platform technology within the rigorous and timely setting of COVID-19, the UVC may portend a novel class of gene and cell therapy prophylaxis for potential future viral pandemics. 


\section{METHODS}

440

441

iPS cell culture, irradiation, and cryopreservation

442 Human iPS cells (Thermo Fisher) were cultured on vitronectin-coated $T 225 \mathrm{~cm}^{2}$ flasks using complete

443 mTesSR Plus medium (StemCell Technologies) supplemented with 1\% penicillin/streptomycin, Rock 444 inhibitor (StemCell Technologies) at 1:1000 dilution. For drug selection, G148 was used at 500ug/ml 445 and puromycin at $5 \mathrm{ug} / \mathrm{ml}$ (Sigma-Aldrich). Cultures were maintained at $37^{\circ} \mathrm{C}, 5 \% \mathrm{CO} 2$ in a humidified 446 incubator. Harvesting of engineered UVC was performed using accutase (StemCell Technologies) and 447 cells were counted using a CellDrop cell counter (DeNovix). Cells were irradiated at a total single dose 448 of $10 \mathrm{~Gy}$, before centrifugation at $300 \mathrm{xg}$ for 10 minutes followed by resuspension in $100 \mu \mathrm{l}$ of 449 CryoStor-CS10 freezing media (StemCell Technologies). The UVC preparations for use in non-human 450 primate studies were analyzed for endotoxin levels (Wickham Laboratories Ltd) and absence of 451 mycoplasma (Mycoplasma Experience Ltd).

452

\section{CRISPR genetic engineering}

454 CRISPR sgRNAs targeting the human B2M gene, PPP1R12C (AAVS1), and the ROSAßgeo26 locus were designed and validated for indel formation at the selected genomic site. Up to 6 sgRNAs per target gene were tested and the most efficient sgRNA was selected containing 2'-O-methyl and $3^{\prime}$ phosphorothioate modifications to the first three $5^{\prime}$ and the last three $3^{\prime}$ nucleotides (Synthego). $2 \times 10^{6}$ UVC cells were electroporated using a Neon Nucleofector (Lonza) in Buffer P3 (Lonza) with Cas9 protein (IDT) precomplexed with sgRNA, in a total volume of $100 \mu$ using electroporation program CM138. Gene targeting vectors carrying an expression cassette for expression of human MICA or the SARS-CoV-2 WA1/2020 spike gene, targeting the Rosa26 and AAVS1 locus respectively, were coelectroporated at $4 \mu \mathrm{g}$. Indels introduced by CRISPR editing were detected by PCR and Sanger sequence using DNA primers designed to amplify a 600-900 base pair region surrounding the sgRNA target site. A minimum of 24 hours after electroporation, genomic DNA was extracted using the DirectPCR Lysis solution (Viagen Biotech) containing Proteinase $\mathrm{K}$ and target regions were amplified by PCR using the GoTaq G2 PCR mastermix (Promega). Correct and unique amplification of the target regions was verified by agarose gel electrophoresis before purifying PCR products using the QIAquick PCR Purification Kit (Qiagen). For analysis by TIDE, PCR amplicons were Sanger sequenced (Eurofins or Genewiz) and paired .ab1 files of control versus edited samples were analyzed using Synthego's ICE tool (https://ice.synthego.com). 
473

\section{Intracellular spike protein staining}

474 Engineered UVC were harvested and then fixed and permeabilized using BD Cytofix/Cytoperm 475 Fixation/Permeabilization Solution (ThermoFisher). Cells were then stained for intracellular spike 476 protein using an Anti-SARS-CoV-2 Spike Glycoprotein S1 antibody (Abcam, ab275759, 1:50) followed 477 by Goat Anti-Rabbit IgG H\&L (Alexa Fluor 488) (Abcam, ab150077, 1:500). Flow analysis was carried 478 out on a Fortessa flow cytometer (BD Bioscience), and data analyzed, and flow cytometry figures generated using FlowJo 10 software (BD Biosciences).

480

Flow cytometry analysis of cell surface antigen expression

482

For flow cytometric analysis of cell surface expression of MHC-I, MICA and SARS-CoV-2 spike protein, cells were harvested from culture plates and washed using PBS with 1\% Bovine Serum Albumen (Thermo Scientific) and were then stained with PE anti-human MICA/MICB Antibody (6D4, Biolegend), Alexa Fluor 647 anti-human HLA-A,B,C (W6/32, Biolegend), and anti-SARS-CoV-2 Spike Glycoprotein S1 antibody (Abcam, ab275759, 1:50) followed by Goat Anti-Rabbit IgG H\&L (Alexa Fluor 488) (Abcam, ab150077, 1:500). Live/Dead Fixable Dead Cell Stains (Invitrogen) were included in all experiments to exclude dead cells. After staining, cells were resuspended in PBS with $2 \%$ Human Heat Inactivated AB Serum (Sigma) and 0.1 M EDTA pH 8.0 (Invitrogen) before analysis on a Fortessa flow cytometer (BD Bioscience) and data analyzed using FlowJo 10 software (BD Biosciences).

491

\section{Western blot}

493

The SARS-CoV-2 spike glycoprotein was detected in UVC lysates by western blotting. Briefly, cells were lysed by RIPA buffer ( $20 \mathrm{mM}$ Tris-HCl pH 7.5, $150 \mathrm{mM} \mathrm{NaCl} 1 \mathrm{mM}$ EDTA, 0.1\% SDS, 1\% NP40, $1 \mathrm{x}$ protease inhibitor cocktail). Samples were spun at $4^{\circ} \mathrm{C}$ for $10 \mathrm{mins}$ at $12,000 \mathrm{xg}$ and the pellet discarded. Protein content was measured using BCA Assay (ThermoFisher) using a PHERAstar plate reader (BMG Labtech) at $560 \mathrm{~nm}$. LDS Sample Buffer was added to $30 \mathrm{ng}$ of protein sample to make a $1 \mathrm{x}$ solution, with $0.5 \mu \mathrm{l}$ of b-mercaptoethanol per well and heated at $70^{\circ} \mathrm{C}$ for 10 minutes before separation on a polyacrylamide gel (Bio-Rad Mini-PROTEAN TGX Gel 4-15\%) and transferred to a PVDF membrane. Membranes were blocked in blocking buffer ( $5 \%$ non-fat powdered milk in TBST), before incubation with primary antibodies in blocking buffer (Rabbit polyclonal anti-SARS-Cov2, Sino Biological 40591-T62, 1:6000 dilution or Mouse b-actin, Abcam 8226, $1 \mu \mathrm{g} / \mathrm{ml}$ ), detected with HRP conjugated secondaries in blocking buffer (Goat anti-Rabbit HRP, Sino Biological SSA003, $0.5 \mu \mathrm{g} / \mathrm{ml}$ or Goat anti-Mouse HRP, Abcam ab205719, 1: 4000 dilution) and visualised using the SuperSignal West Femto kit (ThermoFisher) as per kit instructions. 
507

508

509

510

511

512

513

514

515

516

517

518

519

520

521

522

523

524

525

526

527

528

529

530

531

532

533

534

535

536

537

538

539

540

qPCR measurement of stem cell factors

Total RNA was extracted from UVC cells using the ReliaPrep RNA miniprep (Promega) according to the manufacturer's instructions (a DNase treatment was included for all samples), and RNA concentration and absorbance ratios were measured using a Nanodrop One Spectrophotometer (ThermoFisher). cDNA was synthesized using a High-Capacity cDNA Reverse Transcription Kit (the Applied Biosystems) in a total volume of $20 \mu \mathrm{l}$ to produce DNA that was subsequently assessed by spectrophotometric analysis and diluted to $100 \mathrm{ng} / \mu \mathrm{l}$. Individual master mixes with each of the DNA-primer combinations for detection of human SOX2, NANOG, OCT4, DNC, Vimentin, HES5 and GATA6 genes were made for 3 replicates using the Brilliant III Ultra-Fast SYBR green qPCR master mix (Agilent Technologies) and analyzed on a CFX Opus Real-Time PCR system (BioRad) using the following program: $95^{\circ} \mathrm{C}$ for 15 minutes for $1 \mathrm{cycle} ; 95^{\circ} \mathrm{C}$ for 15 seconds for 40 cycles; $60^{\circ} \mathrm{C}$ for 30 seconds.

\section{SARS-COV-2 spike protein ELISA}

Cell pellets were harvested and lysed in $20 \mu \mathrm{l}$ Cell Extraction Buffer (Invitrogen) containing protease inhibitors (Sigma) on ice for 30 minutes, with 3 brief vortexing every 10 minutes. Samples were centrifuged at $13,000 \mathrm{rpm}$ for 10 minutes at $4^{\circ} \mathrm{C}$ to pellet insoluble contents. S1 Spike protein was detected using a Covid-19 S-protein ELISA kit (Abcam) specific to S1RBD. Samples were diluted to a range determined to be within the working range of the ELISA kit used and the assay procedure was follows as per manufacturer's instructions. The resulting colorimetric signal was detected at $450 \mathrm{~nm}$ using a PHERAstar (BMG LABTECH) plate reader. GraphPad Prism was used to plot a standard curve and interpolate the sample values using a 4-parameter logistic fit.

\section{UVC Proliferation and apoptosis assays}

To quantify apoptosis of UVC post-irradiation, cells were stained using a FITC Annexin V Apoptosis Detection Kit with 7-AAD (Biolegend). Proliferation of cells was measured staining of control and Irradiated UVC with either $2 \mu \mathrm{M}$ Cell Trace Yellow (Abcam) according to kit protocol and analyzing the dilution of the dye at 24-hour periods over 3-days and measuring fluorescence intensity. Flow analysis was carried out on a Fortessa flow cytometer (BD Bioscience), and data analyzed, and flow cytometry figures generated using FlowJo 10 software (BD Biosciences).

\section{CAM cytotoxicity assay}

Both MHC-I expressing and MHC-I deficient (B2M knockout) UVC were used as target cells for NK cell cytotoxicity assay. Trypsinized cells were stained with calcein acetoxymethyl ester (CAM, Invitrogen) at a $10 \mu \mathrm{M}$ concentration for 1 hour at $37^{\circ} \mathrm{C}$ and then washed to remove excess dye. NK cells highly 
541 enriched from normal cynomolgus macaque (Macaca fascicularis) blood samples using a CD3

542 depletion kit (Miltenyi Biotec), were used as effector cells. NK cell effectors and stained target cells 543 were co-cultured in 96 well round bottom plates at effector: target (E:T) ratios of 1:1 and 5:1. Control

544 wells included - only target cells for spontaneous release of CAM and target cells treated with Triton-

$545 \times 100$ for maximum release of CAM. At the end of 4-hour incubation, supernatant was collected for

546 CAM measurement in a fluorescent plate reader at $530 \mathrm{~nm}$. Percent-specific lysis = (test release -

547 spontaneous release)/(maximum release - spontaneous release).

548

549 Nucleofection of NKG2D ligands in iPS cells

550 UVC were cultured in EGM2 (Lonza) media supplemented with 20 ng/mI VEG-F (Peprotech) until 70-

$55190 \%$ confluent, in tissue culture flasks pre-coated with sterile $0.1 \%$ gelatin in PBS for 1 hour at $37^{\circ} \mathrm{C}$.

552 The cells were removed from culture flasks using trypsin, washed, and transfected with plasmid DNA

553 containing either MICA, MICB or ULBP-1 genes after optimizing nucleofection conditions using primary 554 cell 4D nucleofector kit and 4D nucleofector system (Lonza). After 48 hours of culture, transfected 555 cells were stained with aqua dye for live/dead discrimination and corresponding antibodies556 MICA/MICB (Clone 6D4, PE, BioLegend) or ULBP-1 (clone 170818, PE, R \& D Systems). Stained cells 557 were fixed with $2 \%$ paraformaldehyde and acquired on LSRII flow cytometer. Transfection efficiency was calculated as \% live cells expressing transfected protein.

559

560

\section{NK cell intracellular cytokine staining assay}

561 NK cell effectors were enriched from normal cynomolgus macaque (Macaca fascicularis) blood 562 samples using a CD3 depletion Kit (Miltenyi Biotec). Target and effector cells were plated at E:T ratio 563 of 2:1 in a 96 well round bottom plate. Anti-CD107a antibody (clone H4A3, ECD conjugate, BD 564 Biosciences), brefeldin $A$ and monensin (BD Biosciences) were added to all the samples prior to 565 incubation. After 6 hours of incubation at $37^{\circ} \mathrm{C}$, the cells were washed and stained with aqua dye used 566 for live and dead cell discrimination for 20 minutes at room temperature. The cells were then washed 567 and stained for surface markers that included CD3 (SP34.2, BV421, BD Biosciences), CD14 (M5E2, 568 BV650, BD Biosciences), CD16 (3G8, BUV496, BD Biosciences), CD20 (L27, BV570, BD Biosciences), 569 CD56 (NCAM1.2, BV605, BD Biosciences), HLA-DR (G46-6, APC-H7, BD Biosciences) and NKG2A (Z199, 570 PE-Cy7, BD Biosciences) to delineate NK effector cells. Following incubation for 20 minutes, cells were 571 washed and permeabilized using fix \& perm reagent (Thermofisher Scientific) as per manufacturer's 572 recommendation. Intracellular cytokine staining was performed for macrophage inflammatory 573 protein $1 \beta$ (MIP-1 $\beta$; D21-1351, FITC, BD Biosciences) interferon- $\gamma$ (IFN- $\gamma ; B 27, B U V 395, B D$ 574 Biosciences), tumor necrosis factor- $\alpha$ (TNF- $\alpha$; Mab11, BV650, BD Biosciences) at $4^{\circ} \mathrm{C}$ for 15 minutes. 
575 Cells were washed, fixed, and acquired on LSRII flow cytometer. Unstimulated NK cells were used for

576 background subtraction of percent positive cells. NK cells stimulated with leukocyte activation cocktail

577 (BD Biosciences) were used as positive control for the assay.

578

\section{Animals and study design}

580 Outbred adult male and female rhesus macaques ( $M$. mulatta) and cynomolgus macaques ( $M$.

581 fascicularis), 6-12 years old, were randomly allocated to groups. All macaques were housed at

582 Bioqual. Macaques were treated with irradiated UVC at doses of either $1 \times 10^{7}$ or $1 \times 10^{8}$ cells $(n=3-6)$,

583 and sham controls $(n=3-6)$. Prior to immunization, the cryopreserved doses of irradiated UVC were

584 thawed at $37^{\circ} \mathrm{C}$, then $900 \mu \mathrm{l}$ of 1 XPBS was added to each vial of $100 \mu \mathrm{l}$ UVC in Cryostore freezing

585 media. Macaques received a prime immunization of $1 \mathrm{ml}$ of UVC by the intramuscular route without

586 adjuvant at week 0 . At weeks 4 or 6 , macaques received a boost immunization of either $1 \times 10^{7}$ or $1 \times 10^{8}$

587 UVC. At week 10 all macaques were challenged with $1.0 \times 10^{5} \mathrm{TCID}_{50}\left(1.2 \times 10^{8}\right.$ RNA copies, $1.1 \times 10^{4}$

588 PFU) SARS-CoV-2, which was derived from B.1.617.2 (Delta). Viral particle titers were assessed by RT-

589 PCR. Virus was administered as $1 \mathrm{ml}$ by the intranasal route $(0.5 \mathrm{ml}$ in each nare $)$ and $1 \mathrm{ml}$ by the

590 intratracheal route. All immunological and virological assays were performed blinded. All animal

591 studies were conducted in compliance with all relevant local, state, and federal regulations and were

592

approved by the Bioqual Institutional Animal Care and Use Committee (IACUC).

593

594 Subgenomic viral mRNA assay

595 SARS-CoV-2 E gene sgRNA was assessed by RT-PCR using primers and probes as previously described ${ }^{49}$,

$59650,51,52$. In brief, to generate a standard curve, the SARS-CoV-2 E gene sgRNA was cloned into a

597 pcDNA3.1 expression plasmid; this insert was transcribed using an AmpliCap-Max T7 High Yield

598 Message Maker Kit (Cellscript) to obtain RNA for standards. Before RT-PCR, samples collected from

599 challenged macaques or standards were reverse-transcribed using Superscript III VILO (Invitrogen)

600 according to the manufacturer's instructions. A Taqman custom gene expression assay (ThermoFisher

601 Scientific) was designed using the sequences targeting the $E$ gene sgRNA. Reactions were carried out

602 on a QuantStudio 6 and 7 Flex Real-Time PCR System (Applied Biosystems) according to the

603 manufacturer's specifications. Standard curves were used to calculate sgRNA in copies per ml or per

604 swab; the quantitative assay sensitivity was 50 copies per $\mathrm{ml}$ or per swab.

605

606

Serum antibody ELISA

607 RBD-specific binding antibodies were assessed by ELISA as previously described ${ }^{9,10}$. In brief, 96-well 608 plates were coated with $1 \mu \mathrm{g} \mathrm{ml-1}$ SARS-CoV-2 RBD protein (A. Schmidt, MassCPR) in $1 \times$ DPBS and 
incubated at $4{ }^{\circ} \mathrm{C}$ overnight. After incubation, plates were washed once with wash buffer $(0.05 \%$ Tween 20 in 1× DPBS) and blocked with $350 \mu$ l casein block per well for 2-3 hour at room temperature. After incubation, block solution was discarded, and plates were blotted dry. Serial dilutions of heatinactivated serum diluted in casein block were added to wells and plates were incubated for 1 hour at room temperature, before three further washes and a 1-hour incubation with a 1:1,000 dilution of anti-macaque IgG HRP (NIH NHP Reagent Program) at room temperature in the dark. Plates were then washed three times, and $100 \mu$ l of SeraCare KPL TMB SureBlue Start solution was added to each well; plate development was halted by the addition of $100 \mu$ I SeraCare KPL TMB Stop solution per well. The absorbance at $450 \mathrm{~nm}$ was recorded using a VersaMax or Omega microplate reader. ELISA endpoint titers were defined as the highest reciprocal serum dilution that yielded an absorbance $>0.2$. The $\log _{10}$ (endpoint titers) are reported.

620

\section{Pseudovirus neutralization assay}

622 The SARS-CoV-2 pseudovirus expressing a luciferase reporter gene were generated in a similar approach to that previously described ${ }^{9,10,16}$. In brief, the packaging construct psPAX2 (AIDS Resource and Reagent Program), luciferase reporter plasmid pLenti-CMV Puro-Luc (Addgene), and spike protein expressing pcDNA3.1-SARS-CoV-2 SACT were co-transfected into HEK293T cells with calcium phosphate. The supernatants containing the pseudotype viruses were collected 48 hours after transfection; pseudotype viruses were purified by filtration with $0.45-\mu \mathrm{m}$ filter. To determine the neutralization activity of the antisera from vaccinated macaques, HEK293T-hACE2 cells were seeded in 96-well tissue culture plates at a density of $1.75 \times 10^{4}$ cells per well overnight. Twofold serial dilutions of heat-inactivated serum samples were prepared and mixed with $50 \mu$ l of pseudovirus. The mixture was incubated at $37^{\circ} \mathrm{C}$ for 1 hour before adding to HEK293T-hACE2 cells. After 48 hours, cells were lysed in Steady-Glo Luciferase Assay (Promega) according to the manufacturer's instructions. SARS-CoV-2 neutralization titers were defined as the sample dilution at which a $50 \%$ reduction in relative light units was observed relative to the average of the virus control wells.

\section{Statistical Analyses}

637 Statistical differences between two sample groups, where appropriate, were analyzed by a standard 638 Student's two-tailed, non-paired, t-test and between three or more sample groups using two-way or 639 three-way ANOVA using GraphPad Prism 9. Analysis of virological data was performed using two-sided 640 Mann-Whitney tests. Correlations were assessed by two-sided Spearman rank-correlation tests. P

641 values are included in the figures or referred to in the legends where statistical analyses have been 642 carried out. $P$ values of less than 0.05 were considered significant. 
D.B. has a sponsored research collaboration funded by Intima Bioscience. Praesidium Bioscience has

patents filed based on the findings described herein.

Fig. 1: CRISPR genetic engineering of an iPS cell line to create a hyper-immunogenic, self-adjuvanting cellular vaccine. (a), Universal vaccine cell CRISPR genetic engineering strategy to create an apoptotic cellular vehicle for antigen delivery. (b), Representative flow-cytometric analysis showing expression of SARS-CoV-2 WA1/2020 spike protein on the cell surface, and (c), by western blot showing spike protein within UVC whole-cell lysates. (d), ELISA quantification of spike protein released upon UVC lysis. (e), Proportion of apoptotic cells at 24- and 72-hours post-irradiation as measured by 7-AAD staining and flow cytometry. (f) Morphology, observed by light microscopy, of engineered UVC during expansion culture, and when reseeded into culture 24 hours after irradiation, showing apoptosis and cell death. (g) Absence of detectable proliferation of irradiated UVC as determined by CellTrace Yellow proliferation dye staining and measuring the dilution of the dye by flow cytometry over 72-hours. (h) Representative flow-cytometric analysis showing deletion of MHC class-I and overexpression of MICA on the UVC surface by CRISPR engineering. (i) Cell counts showing exponential expansion of live engineered UVC over 21-days in culture. (j) Relative expression of pluripotency and self-renewal genes by UVC and the control iPS cells from they were derived, as measured by quantitative-PCR, showing maintenance of an iPS cell gene expression prolife after genetic engineering and expansion. Error bars represent mean +/- SEM.

Fig. 2: Self-Adjuvancy: Enhanced cytolysis of genetically engineered UVC iPS cells via engineered MHC-I deletion and NK apoptotic ligand expression. (a). CRISPR knockout of B2M and loss of MHC class-I enhances the killing of UVC cells by human primary NK cells, showing equivalent levels of cytolysis seen with the MHC class-I deficient $\mathrm{K} 562$ cell line. (b). A similar elevated cytolysis of MHC deficient UVC cells is observed with macaque NK cells. (c-d) When overexpressed transiently on the UVC, NKG2D family ligands show no elevation in markers of NK cell activation by macaque NK cells, except MICA which significantly elevates levels of macrophage inflammatory protein-1 $\beta$ (MIP-1B). (d) When stably overexpressed on the UVC by CRISPR editing, MICA enhanced the NK cell functional responses as measured by ICS. ${ }^{* * P}<0.01$, Error bars represent mean $+/-$ SEM.

Fig. 3: Humoral immune responses in UVC vaccinated macaques. (a) Macaques received a high WA1/2020 spike expressing UVC prime dose $\left(1 \times 10^{8}\right)$ or low UVC prime dose $\left(1 \times 10^{7}\right)$ at week 0 , and a boost dose matched to that of the prime dose at week 6 . Humoral immune responses were assessed at 2-week intervals up to week-10 and then again at weeks 24 and 26 by (b) spike, (c) RBD-specific binding antibody ELISA, and (d) pseudovirus neutralization assays. (e) In addition to the WA1/2020 SARS-CoV-2 variant, detectible neutralizing antibodies against the B.1.351 (Beta) and B.1.617.2 (Delta) variants were observed in immunized macaques at weeks 24 and 26. Red bars reflect median responses. Dotted lines reflect assay limit of quantification. NAb, neutralizing antibody.

Fig. 4: Viral loads in UVC vaccinated macaques after heterologous SARS-CoV-2 challenge. (a) Rhesus macaques were immunized with $1 \times 10^{8}$ WA1/2020 spike expressing UVC at week 0 and received a boost dose of $1 \times 10^{8}$ matched UVC at week 4. Macaques were then challenged at week 6 by the intranasal and intratracheal routes with $1.0 \times 10^{5} \mathrm{TCID}_{50}$ of SARS-CoV-2 B.1.617.2 (Delta). Log10[sgRNA (copies per $\mathrm{ml}$ )] (limit of quantification 50 copies per $\mathrm{ml}$ ) were assessed, and peak viral loads are shown in (b) bronchoalveolar lavage (BAL) samples, and (c) Nasal swabs (NS), in sham controls and vaccinated 
691

692

693

694

695

696

697

698

699

700

701

702

703

704

705

706

707

708

709

710

711

712

713

714

715

716

717

718

719

720

721

722

723

724

725

726

727

728

729

730

731

732

733

734

735

736

737

738

739

740

macaques after challenge. Viral loads were assessed every 2 days (d-e). Dotted lines reflect assay limit of quantification. NAb, neutralizing antibody.

Fig. 5: (a) Proposed mechanism of action of UVC to replicate natural physiologic immunity and (b) polyvalent UVC design for emerging variants (Omicron). Schematic depiction of the UVC design to deliver a polyvalent SARS-Cov-2 B.1.1.529 Omicron variant vaccine, incorporating the Spike, Nucleocapsid, ORF3a and Membrane viral proteins

Supplementary: (S1) Design of the gene targeting vector to insert the SARS-CoV-2 spike gene into the UVC genome by CRISPR-mediated homology-directed repair. The amino acid sequence of the WA1/2020 Spike protein is shown highlighting the furin cleavage and proline-stabilizing mutations. (S2) Humoral immune responses in WA1/2020 spike expressing UVC vaccinated rhesus macaques prior to challenge with SARS-CoV-2 B.1.617.2 (Delta). Antibody responses were assessed at weeks 0, 2, 4, 6 and 8 by pseudovirus neutralization assay and spike specific antibody ELISA. Red bars reflect median responses. Dotted lines reflect assay limit of quantification. NAb, neutralizing antibody.

\section{REFERENCES}

1. Polack, F.P. et al. Safety and Efficacy of the BNT162b2 mRNA Covid-19 Vaccine. N EnglJ Med 383, 2603-2615 (2020).

2. Baden, L.R. et al. Efficacy and Safety of the mRNA-1273 SARS-CoV-2 Vaccine. N Engl J Med 384, 403-416 (2021).

3. Sadoff, J. et al. Safety and Efficacy of Single-Dose Ad26.COV2.S Vaccine against Covid-19. $N$ Engl J Med 384, 2187-2201 (2021).

4. Levin, E.G. et al. Waning Immune Humoral Response to BNT162b2 Covid-19 Vaccine over 6 Months. N Engl J Med 385, e84 (2021).

5. Naaber, P. et al. Dynamics of antibody response to BNT162b2 vaccine after six months: a longitudinal prospective study. Lancet Reg Health Eur 10, 100208 (2021).

6. Thomas, S.J. et al. Safety and Efficacy of the BNT162b2 mRNA Covid-19 Vaccine through 6 Months. N Engl J Med 385, 1761-1773 (2021).

7. Cohn, B.A., Cirillo, P.M., Murphy, C.C., Krigbaum, N.Y. \& Wallace, A.W. SARS-CoV-2 vaccine protection and deaths among US veterans during 2021. Science, eabm0620 (2021).

8. Collier, A.Y. et al. Differential Kinetics of Immune Responses Elicited by Covid-19 Vaccines. $N$ Engl J Med 385, 2010-2012 (2021).

9. Barouch, D.H. et al. Durable Humoral and Cellular Immune Responses 8 Months after Ad26.COV2.S Vaccination. N Engl J Med 385, 951-953 (2021).

10. Planas, D. et al. Reduced sensitivity of SARS-CoV-2 variant Delta to antibody neutralization. Nature 596, 276-280 (2021).

11. Callaway, E. Heavily mutated Omicron variant puts scientists on alert. Nature 600, 21 (2021). 
bioRxiv preprint doi: https://doi.org/10.1101/2021.12.28.474336; this version posted December 29, 2021. The copyright holder for this preprint (which was not certified by peer review) is the author/funder, who has granted bioRxiv a license to display the preprint in perpetuity. It is made available under aCC-BY-NC-ND 4.0 International license.

12. Lefevre, B. et al. Beta SARS-CoV-2 variant and BNT162b2 vaccine effectiveness in long-term care facilities in France. Lancet Healthy Longev 2, e685-e687 (2021).

13. Abu-Raddad, L.J., Chemaitelly, H., Butt, A.A. \& National Study Group for, C.-V. Effectiveness of the BNT162b2 Covid-19 Vaccine against the B.1.1.7 and B.1.351 Variants. N Engl J Med 385, 187-189 (2021).

14. Villarreal, R. \& Casale, T.B. Commonly Used Adjuvant Human Vaccines: Advantages and Side Effects. J Allergy Clin Immunol Pract 8, 2953-2957 (2020).

15. Lauer, K.B., Borrow, R. \& Blanchard, T.J. Multivalent and Multipathogen Viral Vector Vaccines. Clin Vaccine Immunol 24 (2017).

16. Schlingmann, B., Castiglia, K.R., Stobart, C.C. \& Moore, M.L. Polyvalent vaccines: Highmaintenance heroes. PLoS Pathog 14, e1006904 (2018).

17. Pardi, N., Hogan, M.J., Porter, F.W. \& Weissman, D. mRNA vaccines - a new era in vaccinology. Nat Rev Drug Discov 17, 261-279 (2018).

18. Pulendran, B., P, S.A. \& O'Hagan, D.T. Emerging concepts in the science of vaccine adjuvants. Nat Rev Drug Discov 20, 454-475 (2021).

19. Yousefi Avarvand, A., Meshkat, Z., Khademi, F. \& Tafaghodi, M. Immunogenicity of HspX/EsxS fusion protein of Mycobacterium tuberculosis along with ISCOMATRIX and PLUSCOM nanoadjuvants after subcutaneous administration in animal model. Microb Pathog 154, 104842 (2021).

20. Cebon, J.S. et al. Results of a randomized, double-blind phase II clinical trial of NY-ESO-1 vaccine with ISCOMATRIX adjuvant versus ISCOMATRIX alone in participants with high-risk resected melanoma. J Immunother Cancer 8 (2020).

21. Baz Morelli, A. et al. ISCOMATRIX: a novel adjuvant for use in prophylactic and therapeutic vaccines against infectious diseases. J Med Microbiol 61, 935-943 (2012).

22. O'Hagan, D.T., Lodaya, R.N. \& Lofano, G. The continued advance of vaccine adjuvants - 'we can work it out'. Semin Immunol 50, 101426 (2020).

23. Zhou, F., Hansen, L., Pedersen, G., Grodeland, G. \& Cox, R. Matrix M Adjuvanted H5N1 Vaccine Elicits Broadly Neutralizing Antibodies and Neuraminidase Inhibiting Antibodies in Humans That Correlate With In Vivo Protection. Front Immunol 12, 747774 (2021).

24. Bengtsson, K.L. et al. Matrix-M adjuvant enhances antibody, cellular and protective immune responses of a Zaire Ebola/Makona virus glycoprotein (GP) nanoparticle vaccine in mice. Vaccine 34, 1927-1935 (2016).

25. Pavord, S. et al. Clinical Features of Vaccine-Induced Immune Thrombocytopenia and Thrombosis. N Engl J Med 385, 1680-1689 (2021).

26. Long, B., Bridwell, R. \& Gottlieb, M. Thrombosis with thrombocytopenia syndrome associated with COVID-19 vaccines. Am J Emerg Med 49, 58-61 (2021). 
27. Cattaneo, M. Thrombosis with Thrombocytopenia Syndrome associated with viral vector COVID-19 vaccines. Eur J Intern Med 89, 22-24 (2021).

28. Afkhami, S., Yao, Y. \& Xing, Z. Methods and clinical development of adenovirus-vectored vaccines against mucosal pathogens. Mol Ther Methods Clin Dev 3, 16030 (2016).

29. Torgovnick, J. Effectiveness of Covid-19 Vaccines against the B.1.617.2 (Delta) Variant. N Engl J Med 385, e92 (2021).

30. Grant, R. et al. Impact of SARS-CoV-2 Delta variant on incubation, transmission settings and vaccine effectiveness: Results from a nationwide case-control study in France. Lancet Reg Health Eur, 100278 (2021).

31. Fowlkes, A. et al. Effectiveness of COVID-19 Vaccines in Preventing SARS-CoV-2 Infection Among Frontline Workers Before and During B.1.617.2 (Delta) Variant Predominance - Eight U.S. Locations, December 2020-August 2021. MMWR Morb Mortal Wkly Rep 70, 1167-1169 (2021).

32. Abu-Dawud, R., Graffmann, N., Ferber, S., Wruck, W. \& Adjaye, J. Pluripotent stem cells: induction and self-renewal. Philos Trans R Soc Lond B Biol Sci 373 (2018).

33. Deinsberger, J., Reisinger, D. \& Weber, B. Global trends in clinical trials involving pluripotent stem cells: a systematic multi-database analysis. NPJ Regen Med 5, 15 (2020).

34. Shi, Y., Inoue, H., Wu, J.C. \& Yamanaka, S. Induced pluripotent stem cell technology: a decade of progress. Nat Rev Drug Discov 16, 115-130 (2017).

35. Kirchdoerfer, R.N. et al. Pre-fusion structure of a human coronavirus spike protein. Nature 531, 118-121 (2016).

36. Pallesen, J. et al. Immunogenicity and structures of a rationally designed prefusion MERS-CoV spike antigen. Proc Natl Acad Sci U S A 114, E7348-E7357 (2017).

37. Wrapp, D. et al. Cryo-EM Structure of the 2019-nCoV Spike in the Prefusion Conformation. bioRxiv (2020).

38. Szondy, Z., Sarang, Z., Kiss, B., Garabuczi, E. \& Koroskenyi, K. Anti-inflammatory Mechanisms Triggered by Apoptotic Cells during Their Clearance. Front Immunol 8, 909 (2017).

39. Brandstadter, J.D. \& Yang, Y. Natural killer cell responses to viral infection. J Innate Immun 3, 274-279 (2011).

40. Waggoner, S.N. et al. Roles of natural killer cells in antiviral immunity. Curr Opin Virol 16, 1523 (2016).

41. Koutsakos, M. et al. Downregulation of MHC Class I Expression by Influenza A and B Viruses. Front Immunol 10, 1158 (2019).

42. Schuren, A.B., Costa, A.I. \& Wiertz, E.J. Recent advances in viral evasion of the MHC Class I processing pathway. Curr Opin Immunol 40, 43-50 (2016). 
43. Paul, S. \& Lal, G. The Molecular Mechanism of Natural Killer Cells Function and Its Importance in Cancer Immunotherapy. Front Immunol 8, 1124 (2017).

44. Wensveen, F.M., Jelencic, V. \& Polic, B. NKG2D: A Master Regulator of Immune Cell Responsiveness. Front Immunol 9, 441 (2018).

45. Chatterjee, I. et al. Induced Pluripotent Stem (iPS) Cell Culture Methods and Induction of Differentiation into Endothelial Cells. Methods Mol Biol 1357, 311-327 (2016).

46. Rivera, T., Zhao, Y., Ni, Y. \& Wang, J. Human-Induced Pluripotent Stem Cell Culture Methods Under cGMP Conditions. Curr Protoc Stem Cell Biol 54, e117 (2020).

47. Swaidan, N.T. et al. Identification of potential transcription factors that enhance human iPSC generation. Sci Rep 10, 21950 (2020).

48. Shi, G. \& Jin, Y. Role of Oct4 in maintaining and regaining stem cell pluripotency. Stem Cell Res Ther 1, 39 (2010).

49. Mercado, N.B. et al. Single-shot Ad26 vaccine protects against SARS-CoV-2 in rhesus macaques. Nature 586, 583-588 (2020).

50. Yang, Z.Y. et al. A DNA vaccine induces SARS coronavirus neutralization and protective immunity in mice. Nature 428, 561-564 (2004).

51. Yu, J. et al. DNA vaccine protection against SARS-CoV-2 in rhesus macaques. Science $369,806-$ 811 (2020).

52. Chandrashekar, A. et al. SARS-CoV-2 infection protects against rechallenge in rhesus macaques. Science 369, 812-817 (2020).

53. Wolfel, R. et al. Virological assessment of hospitalized patients with COVID-2019. Nature 581, 465-469 (2020).

54. Vogel, A.B. et al. BNT162b vaccines protect rhesus macaques from SARS-CoV-2. Nature 592, 283-289 (2021).

55. Corbett, K.S. et al. Evaluation of the mRNA-1273 Vaccine against SARS-CoV-2 in Nonhuman Primates. N Engl J Med 383, 1544-1555 (2020).

56. Wang, L. \& Cheng, G. Sequence analysis of the Emerging Sars-CoV-2 Variant Omicron in South Africa. J Med Virol (2021).

57. Team, C.C.-R. SARS-CoV-2 B.1.1.529 (Omicron) Variant - United States, December 1-8, 2021. MMWR Morb Mortal Wkly Rep 70, 1731-1734 (2021).

58. Huang, C. et al. Clinical features of patients infected with 2019 novel coronavirus in Wuhan, China. Lancet 395, 497-506 (2020).

59. Bjorkstrom, N.K., Strunz, B. \& Ljunggren, H.G. Natural killer cells in antiviral immunity. Nat Rev Immunol (2021). 
60. Tartof, S.Y. et al. Effectiveness of mRNA BNT162b2 COVID-19 vaccine up to 6 months in a large integrated health system in the USA: a retrospective cohort study. Lancet 398, 1407-1416 (2021).

61. Smits, V.A.J. et al. The Nucleocapsid protein triggers the main humoral immune response in COVID-19 patients. Biochem Biophys Res Commun 543, 45-49 (2021).

62. Vidal, S.J. et al. Correlates of Neutralization against SARS-CoV-2 Variants of Concern by Early Pandemic Sera. J Virol 95, e0040421 (2021).

63. Lu, S. et al. The immunodominant and neutralization linear epitopes for SARS-CoV-2. Cell Rep 34, 108666 (2021).

64. Joyce, M.G. et al. A SARS-CoV-2 ferritin nanoparticle vaccine elicits protective immune responses in nonhuman primates. Sci Transl Med, eabi5735 (2021). 
Fig. 1. CRISPR genetic engineering of an iPS cell line to create a hyper-immunogenic, selfadjuvanting cellular vaccine

a

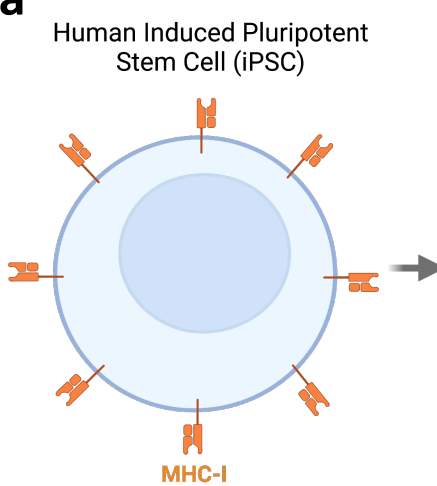

b

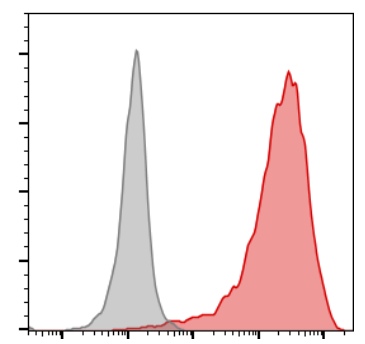

Sars-CoV-2 Spike

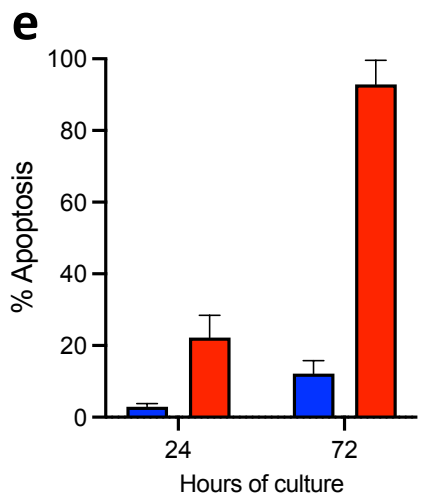

g

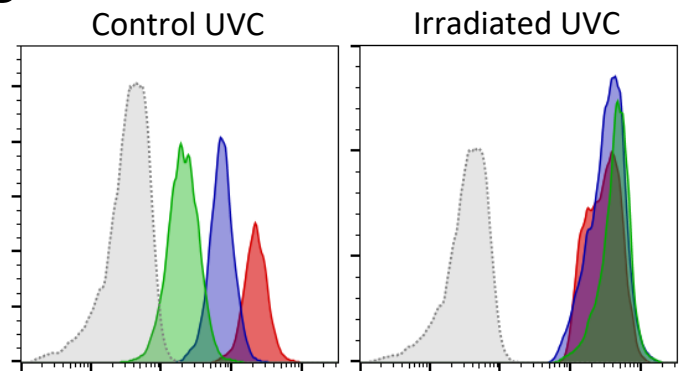

CellTrace Yellow

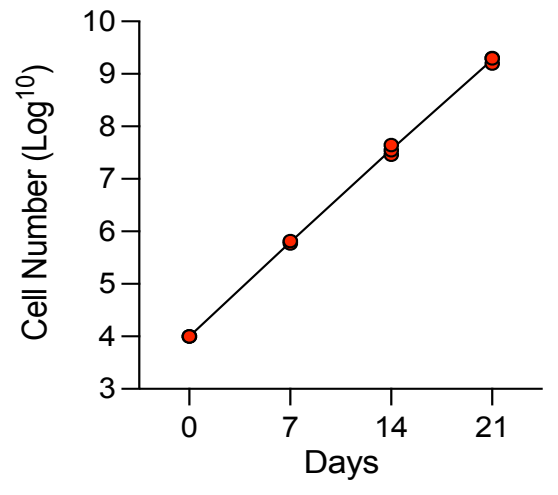

C

f
Universal Vaccine Cell (UVC)
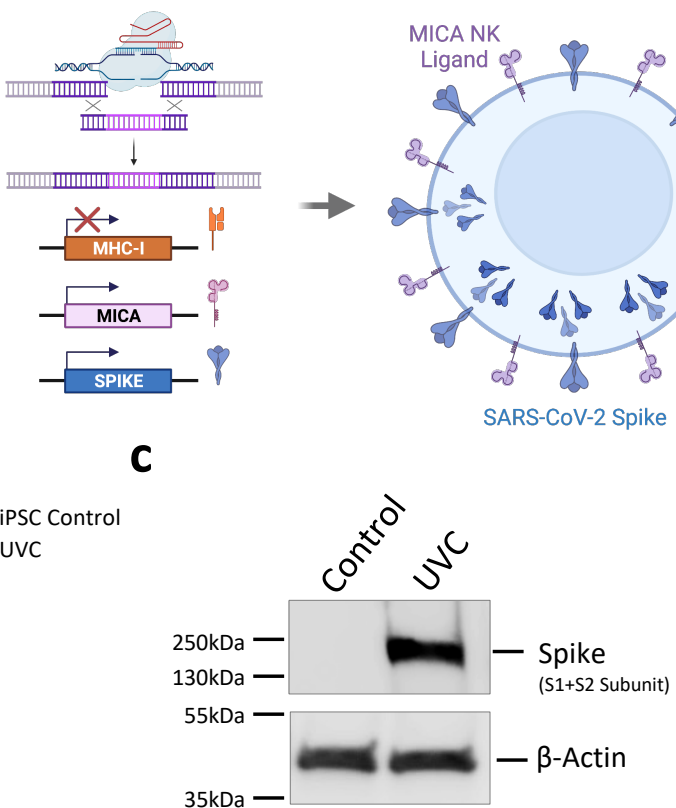

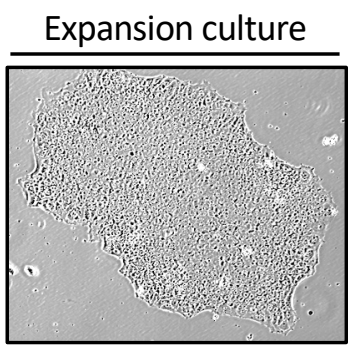

Control UVC

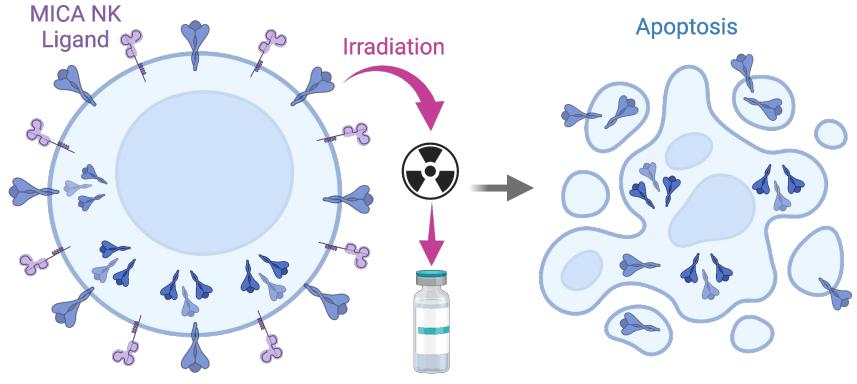

d

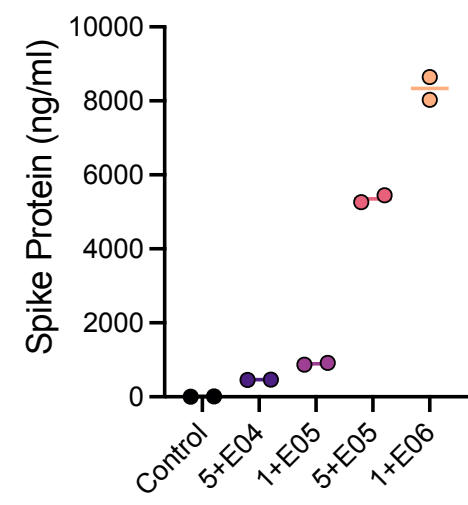

UVC Cell Number

24 hours after irradiation

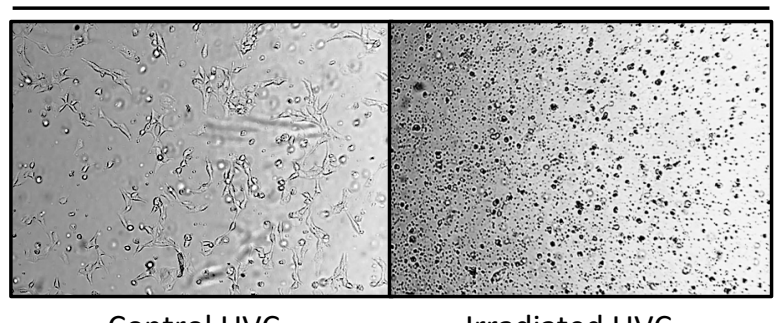

h

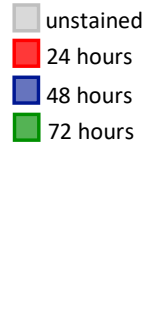

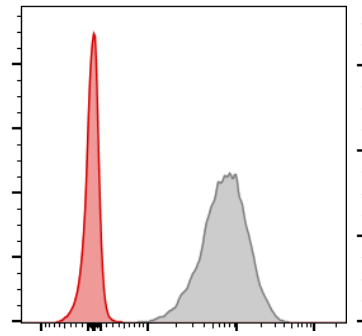

MHC-I (HLA A/B/C)

j

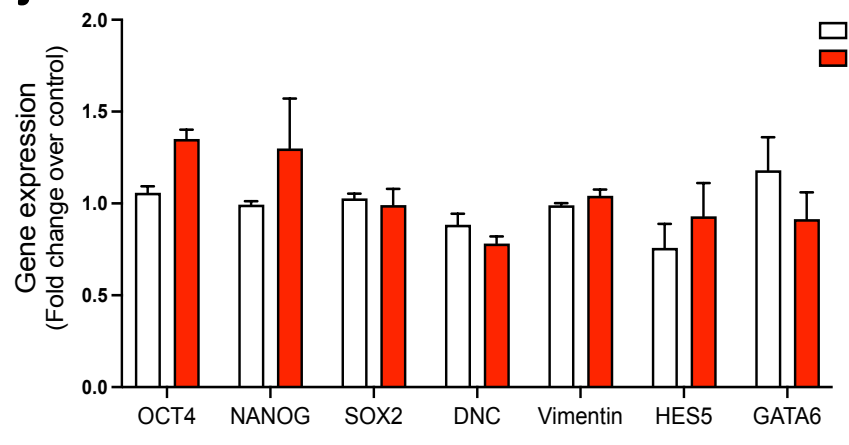


Fig. 2. Self-Adjuvancy: Increased cytolysis of genetically engineered UVC iPS cells via engineered MHC-I deletion and NK apoptotic ligand expression.

a

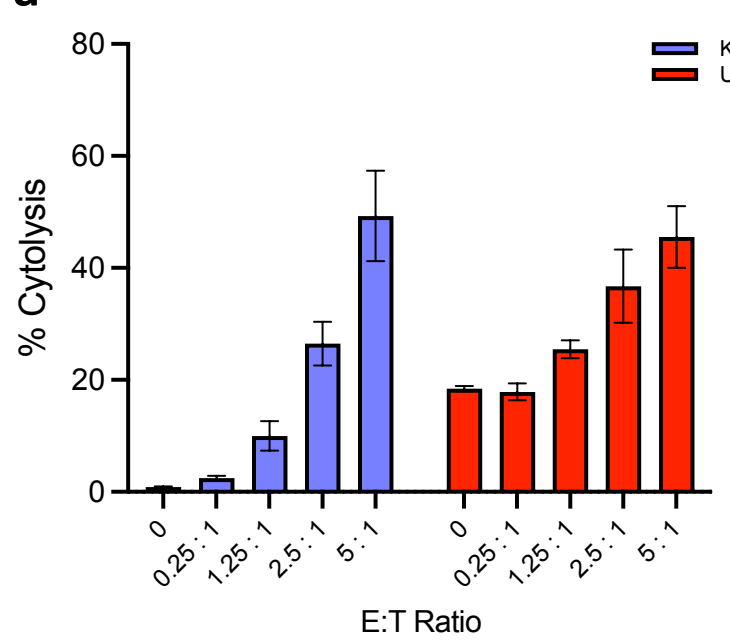

C

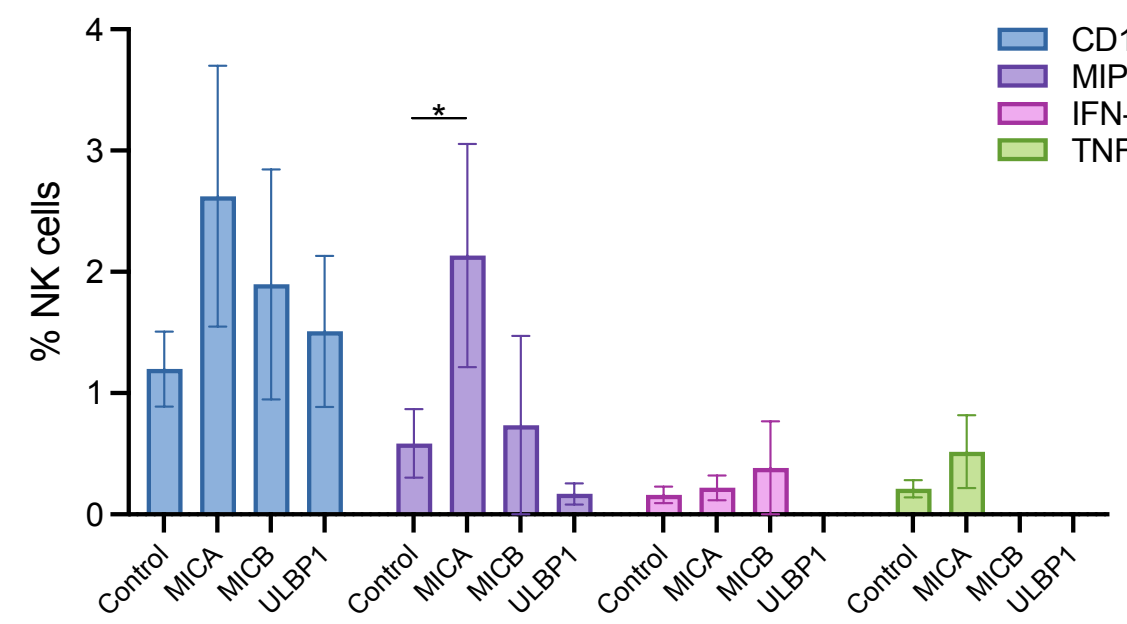

b

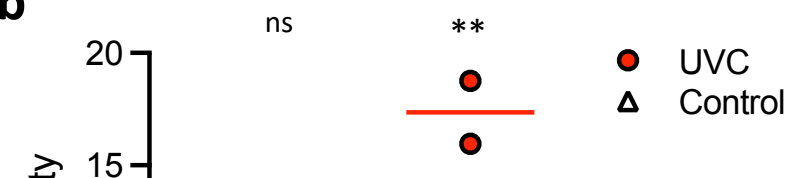

d

Total Responding NK

CD107a
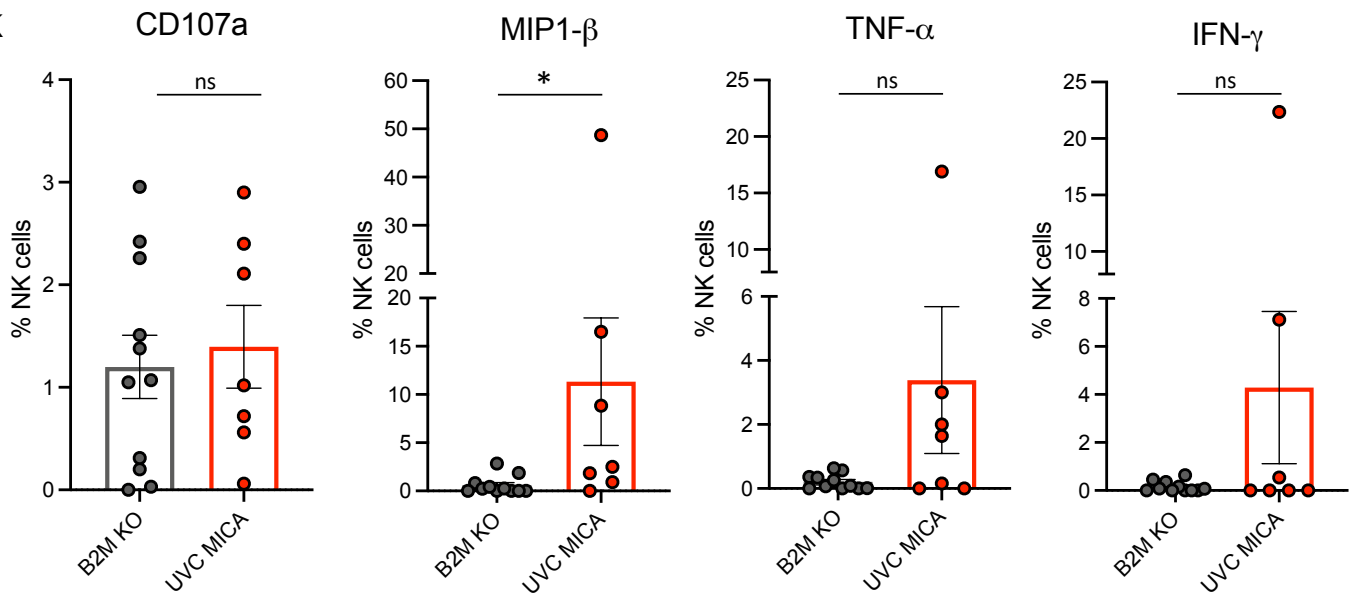
Fig. 3. Humoral immune responses in UVC vaccinated macaques.

a

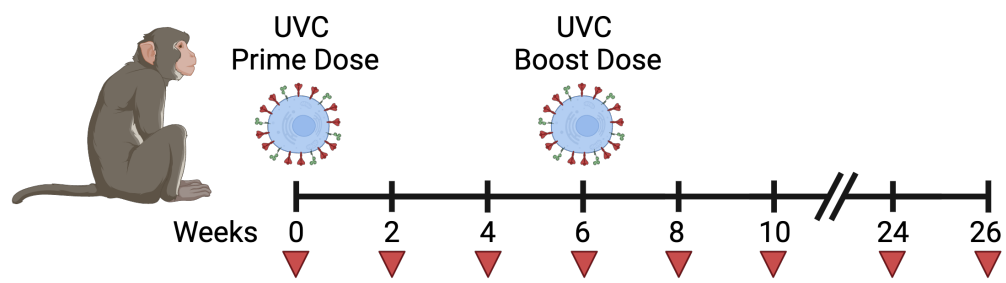

PFU assay; IFNY ELISPOT; NAb and Spike Ab ELISA;

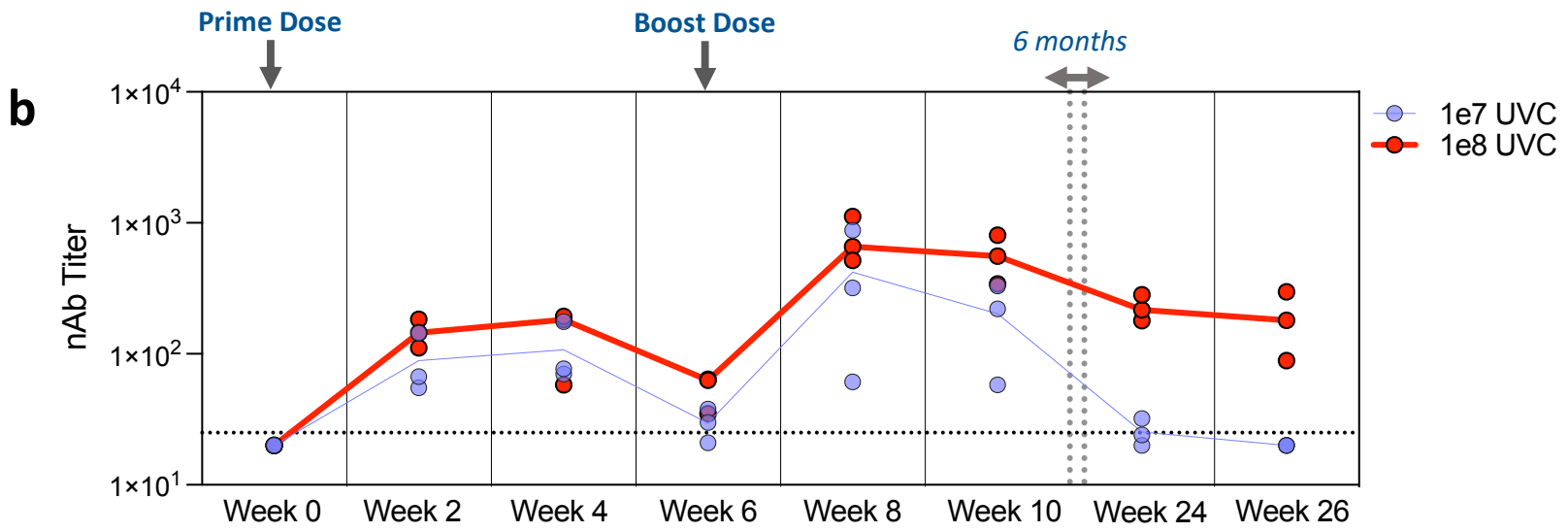

C

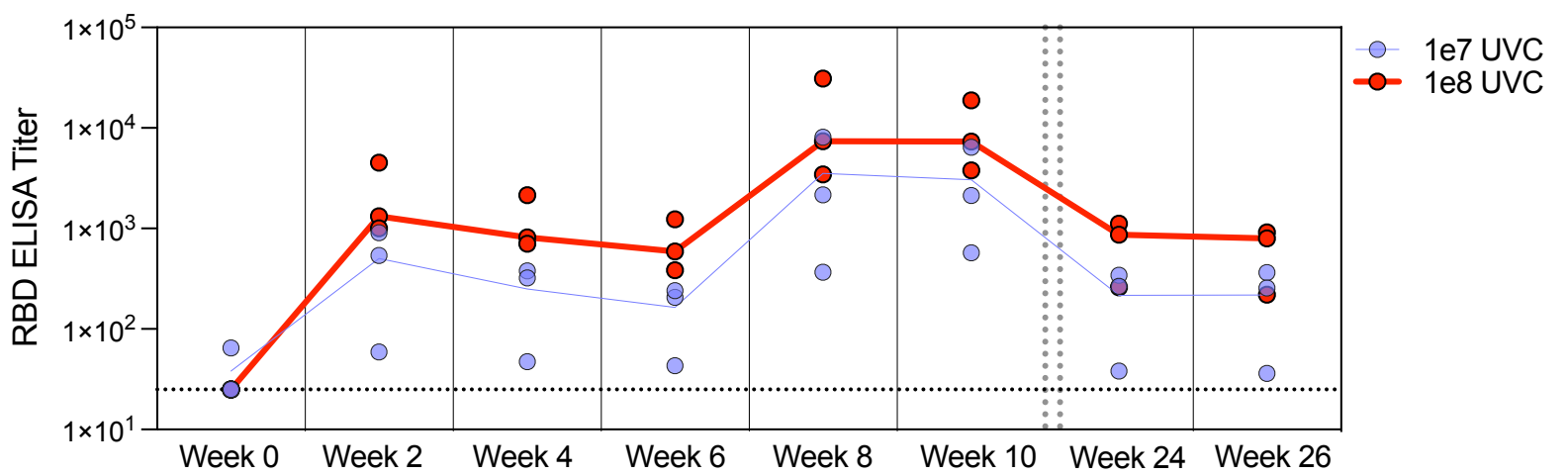

d

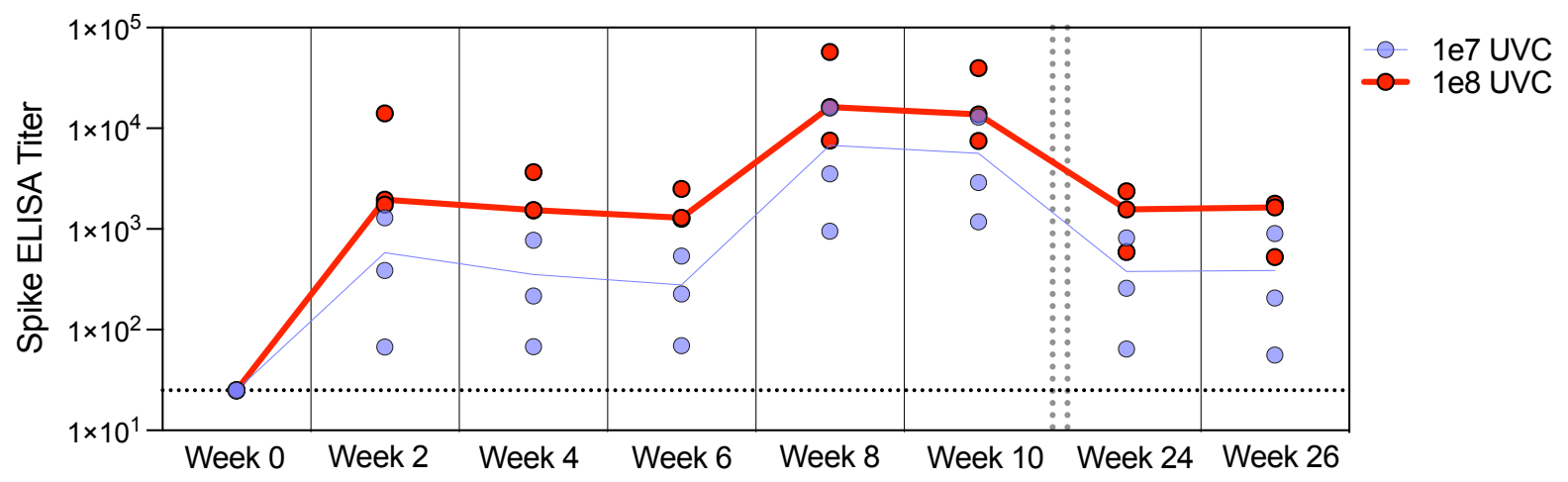

e

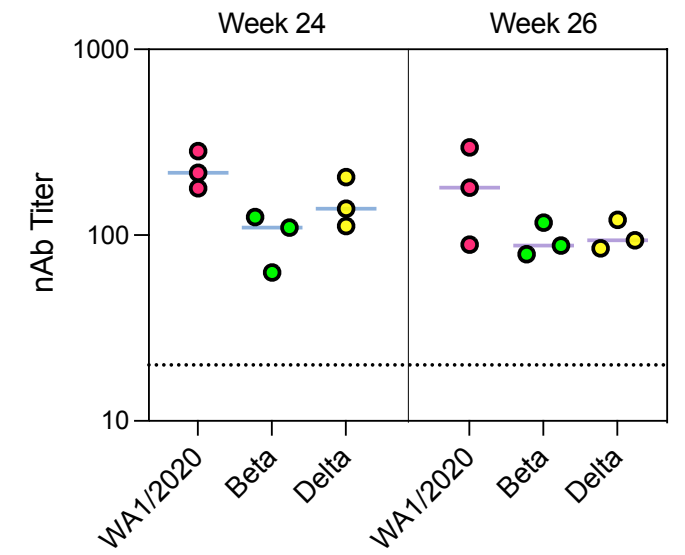


Fig. 4. Viral loads in UVC vaccinated macaques after heterologous SARS-CoV-2 challenge.

a

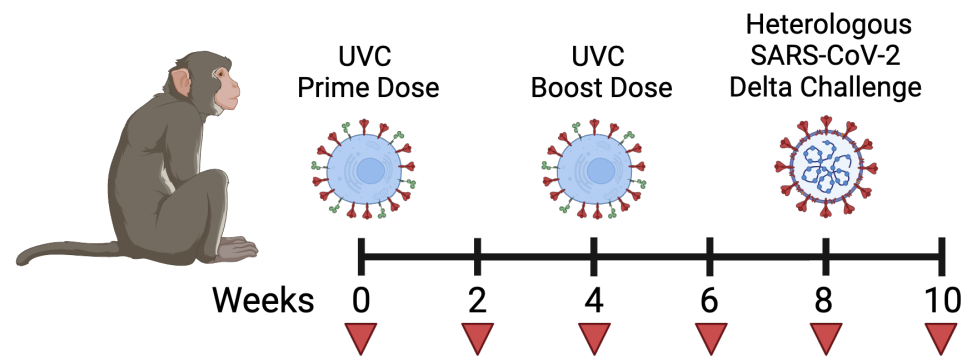

PFU assay; IFNy ELISPOT; NAb and Spike Ab ELISA;
Analysis every 48-hours over 10-days post viral challenge

Viral loads in BAL and nasal swabs

assessed by RT-PCR specific for

subgenomic mRNA (sgRNA) b

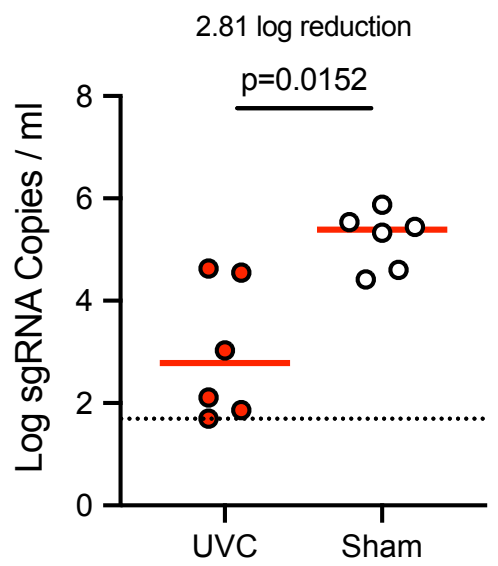

d

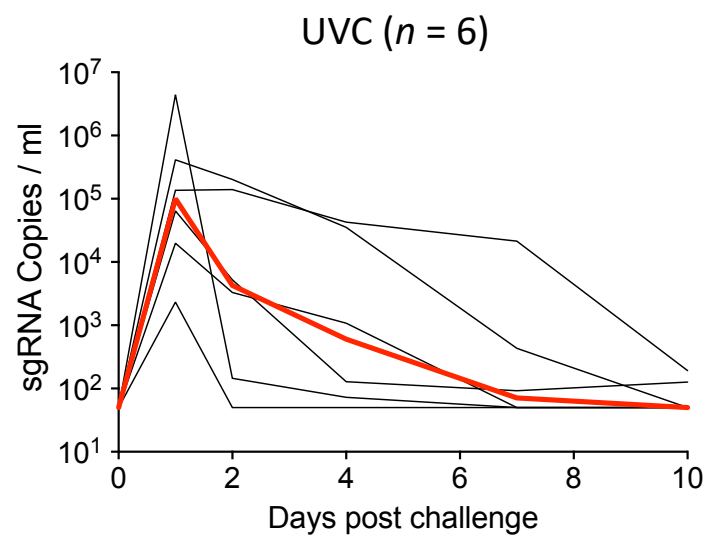

C

NS

0.96 log reduction

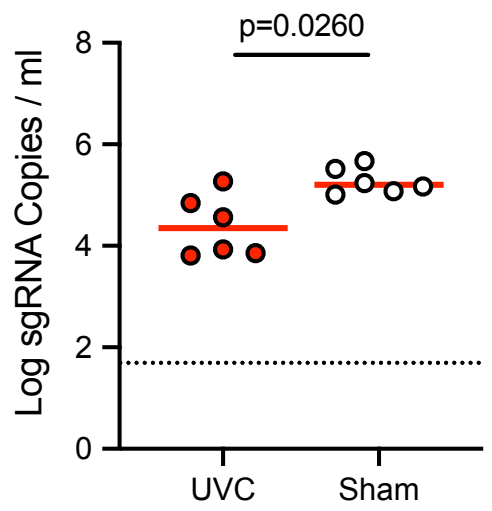

BAL

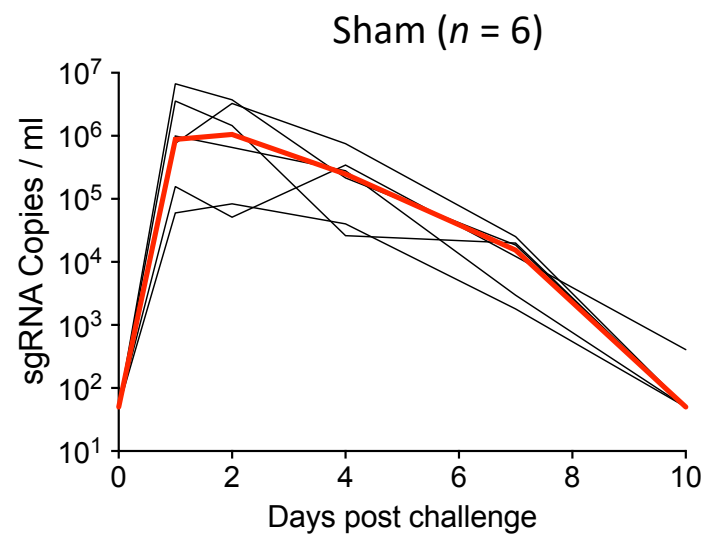

e

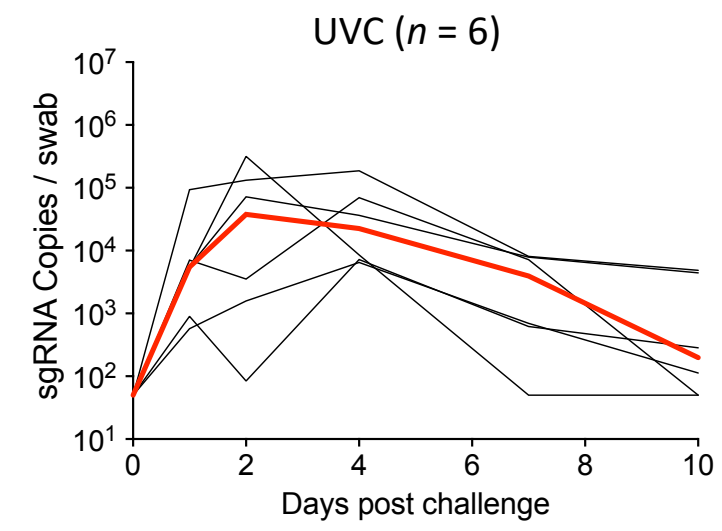

\section{Nasal Swab}

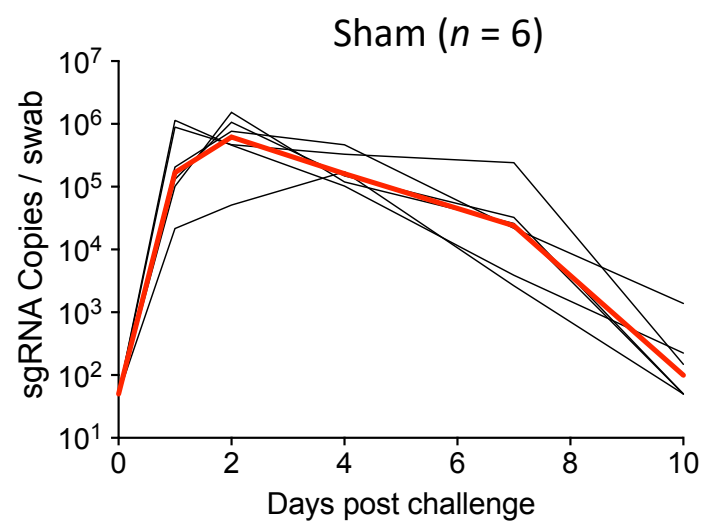


Fig. 5. Proposed mechanism of action of UVC to replicate natural physiologic immunity and poly-valent UVC design for emerging variants (Omicron).

a

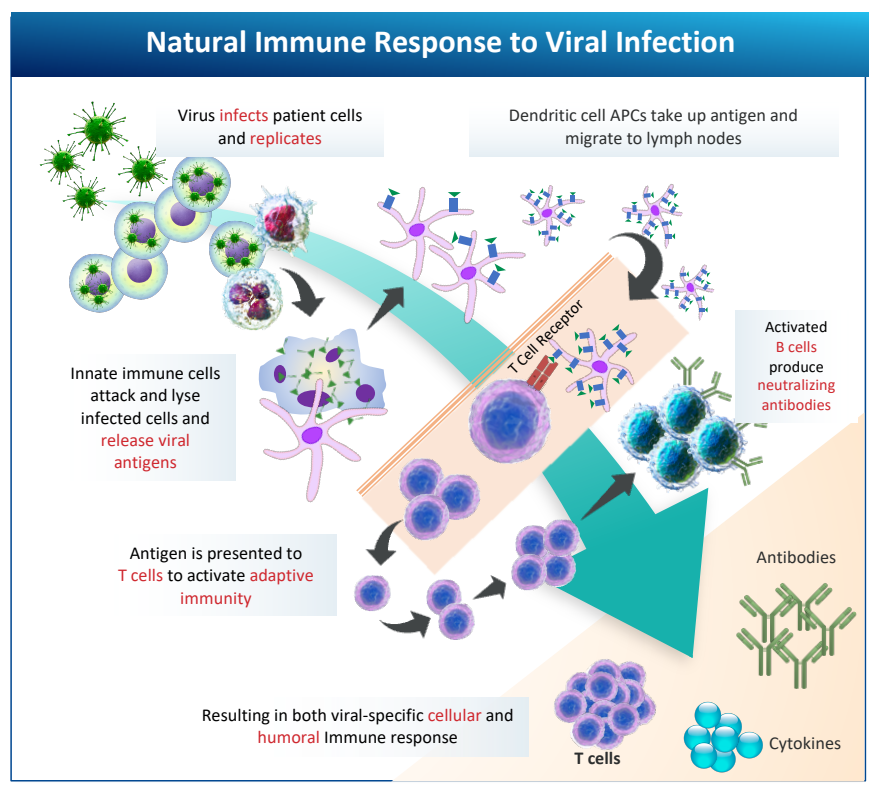

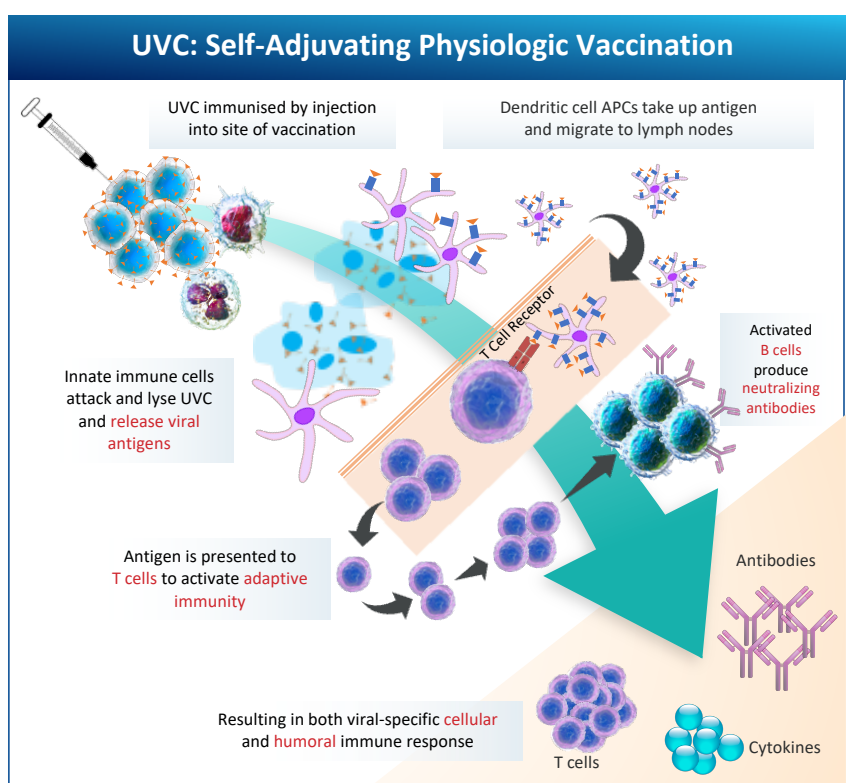

b Genomic organization of SARS-CoV-2
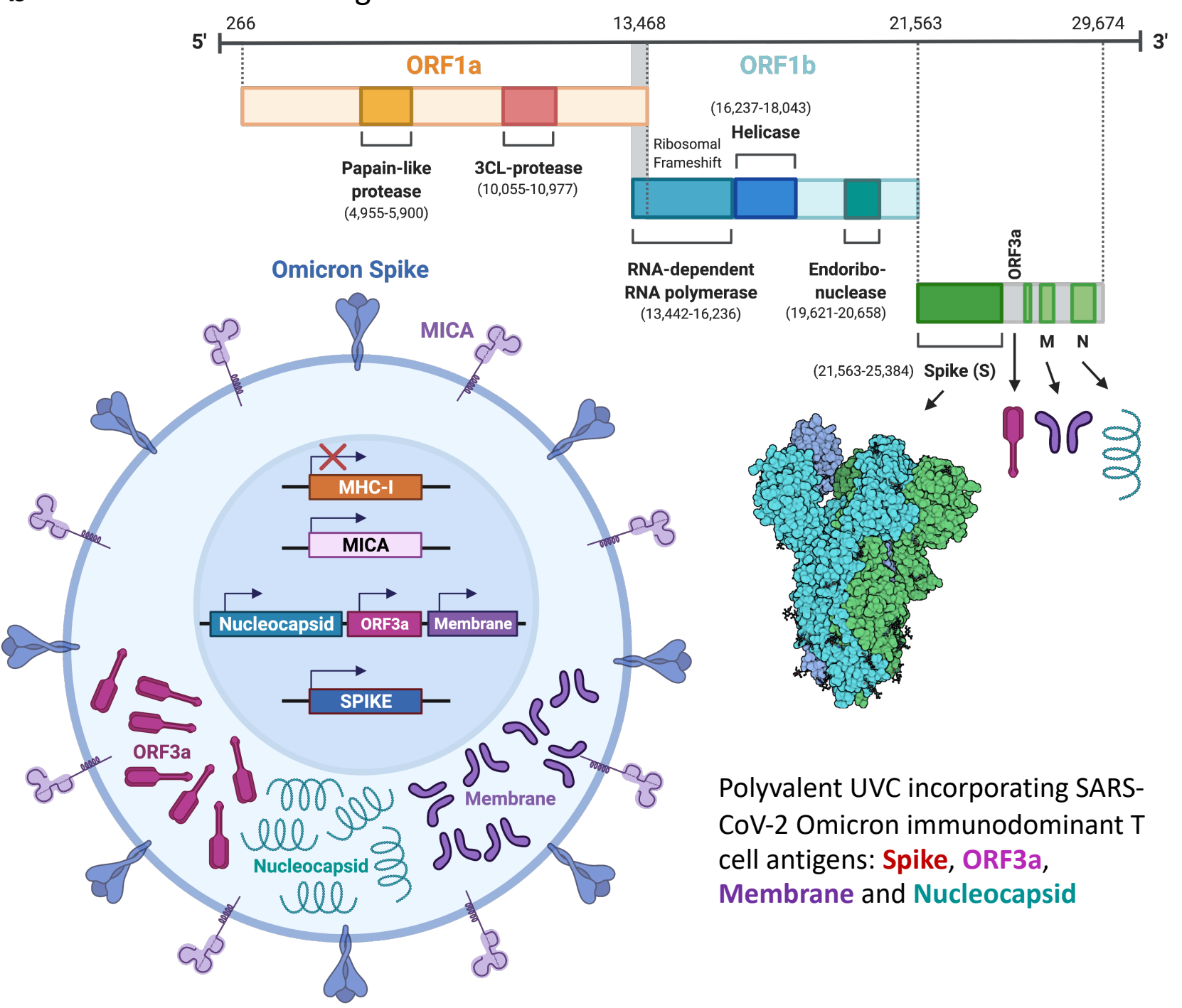

Polyvalent UVC incorporating SARSCoV-2 Omicron immunodominant $\mathrm{T}$ cell antigens: Spike, ORF3a, Membrane and Nucleocapsid 


\section{Supplementary}

S1

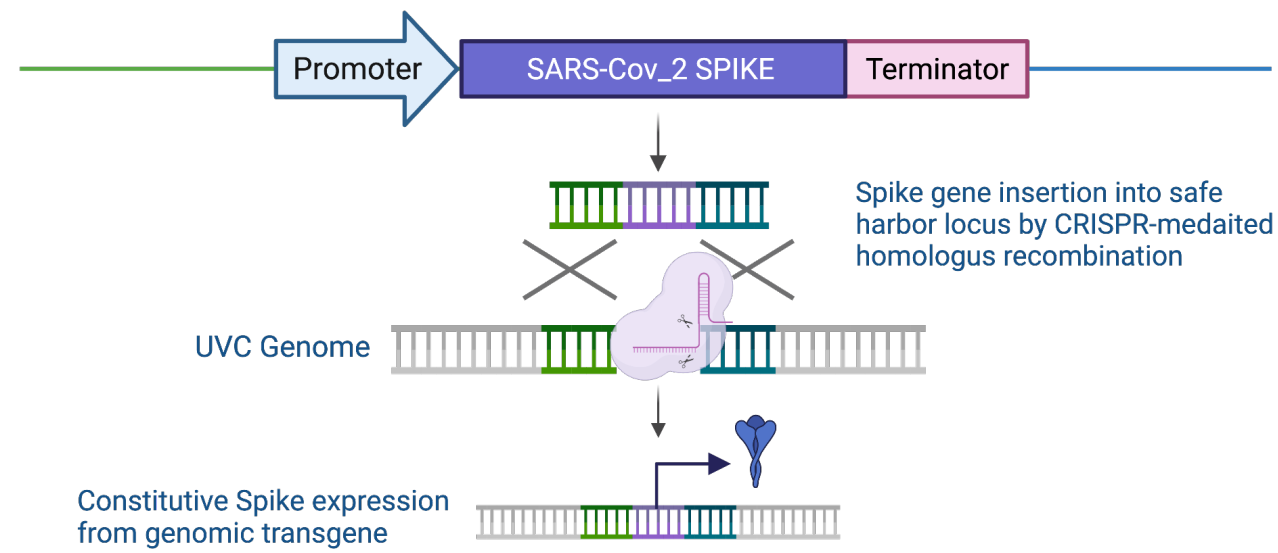

Severe acute respiratory syndrome coronavirus 2 isolate Wuhan-Hu-1 Spike

MAFVFLVLLPLVSSQCVNLTTRTQLPPAYTNSFTRGVYYPDKVFRSSVLHSTQDLFLPFFSNVTWFHAIH VSGTNGTKRFDNPVLPFNDGVYFASTEKSNIIRGWIFGTTLDSKTQSLLIVNNATNVVIKVCEFQFCNDP FLGVYYHKNNKSWMESEFRVYSSANNCTFEYVSQPFLMDLEGKQGNFKNLREFVFKNIDGYFKIYSKHT PINLVRDLPQGFSALEPLVDLPIGINITRFQTLLALHRSYLTPGDSSSGWTAGAAAYYVGYLOPRTFLLKYN ENGTITDAVDCALDPLSETKCTLKSFTVEKGIYQTSNFRVOPTESIVRFPNITNLCPFGEVFNATRFASVYA WNRKRISNCVADYSVIYNSASFSTFKCYGVSPTKLNDLCFTNVYADSFVIRGDEVROIAPGQTGKIADYN YKLPDDFTGCVIAWNSNNLDSKVGGNYNYLYRLFRKSNLKPFERDISTEIYQAGSTPCNGVEGFNCYFPL YKLPDDFTGCVIAWN QSYGFQPTNGVGYQYRVVLSFELLHAPATVCGPKKSTNLVKNKCVNFNFNGLTGTGVLTESNKKFLP FQQFGRDIADTTDAVRDPQTLEILDITPCSFGGVSVITPGTNTSNQVAVLYQDVNCTEVPVAIHADQLTP TWRVYSTGSNVFQTRAGCLIGAEHVNNSYECDIPIGAGICASYQTQTNSPGSASSVASOSIIAYTMSLGA ENSVAYSNNSIAIPTNFTISVTTEILPVSMTKTSVDCTMYICGDSTECSNLLLQYGSFCTQLNRALTGIAVE QDKNTQEVFAQVKQIYKTPPIKDFGGFNFSQILPDPSKPSKRSFIEDLLFNKVTLADAGFIKQYGDCLGDI AARDLICAQKFNGLTVLPPLLTDEMIAQYTSALLAGTITSGWTFGAGAALQIPFAMQMAYRFNGIGVTQ NVLYENQKLIANQFNSAIGKIQDSLSSTASALGKLQDVVNQNAQALNTLVKQLSSNFGAISSVLNDILSRL DPPEAEVQIDRLITGRLOSLOTYVTOQLIRAAEIRASANLAATKMSECVLGOSKRVDFCGKGYHLMSFP DSEAEVQVRLITGTYVAOEKNFTTAPAICHDGKAHFPREGVFSNGTHWFVTORNFYEPQIITTDNTF QSAPHGVVELVTYVPAQEKNFTAPACHDGKAHFPREGVFVSNGTHWFVQRNFYEPQIITIDNTF NLNESLIDLQELGKYEQYIKWPWYIWLGFIAGLIAIVMVTIMLCCMTSCCSCLKGCCSCGSCCKFDEDDS EPVLKGVKLHYTSG*

Furin Clevage Mutation RRAR > GSAS

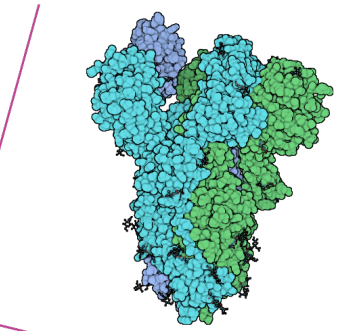

Proline Stablization Mutation $\mathrm{KV}>\mathrm{PP}$

\section{S2}
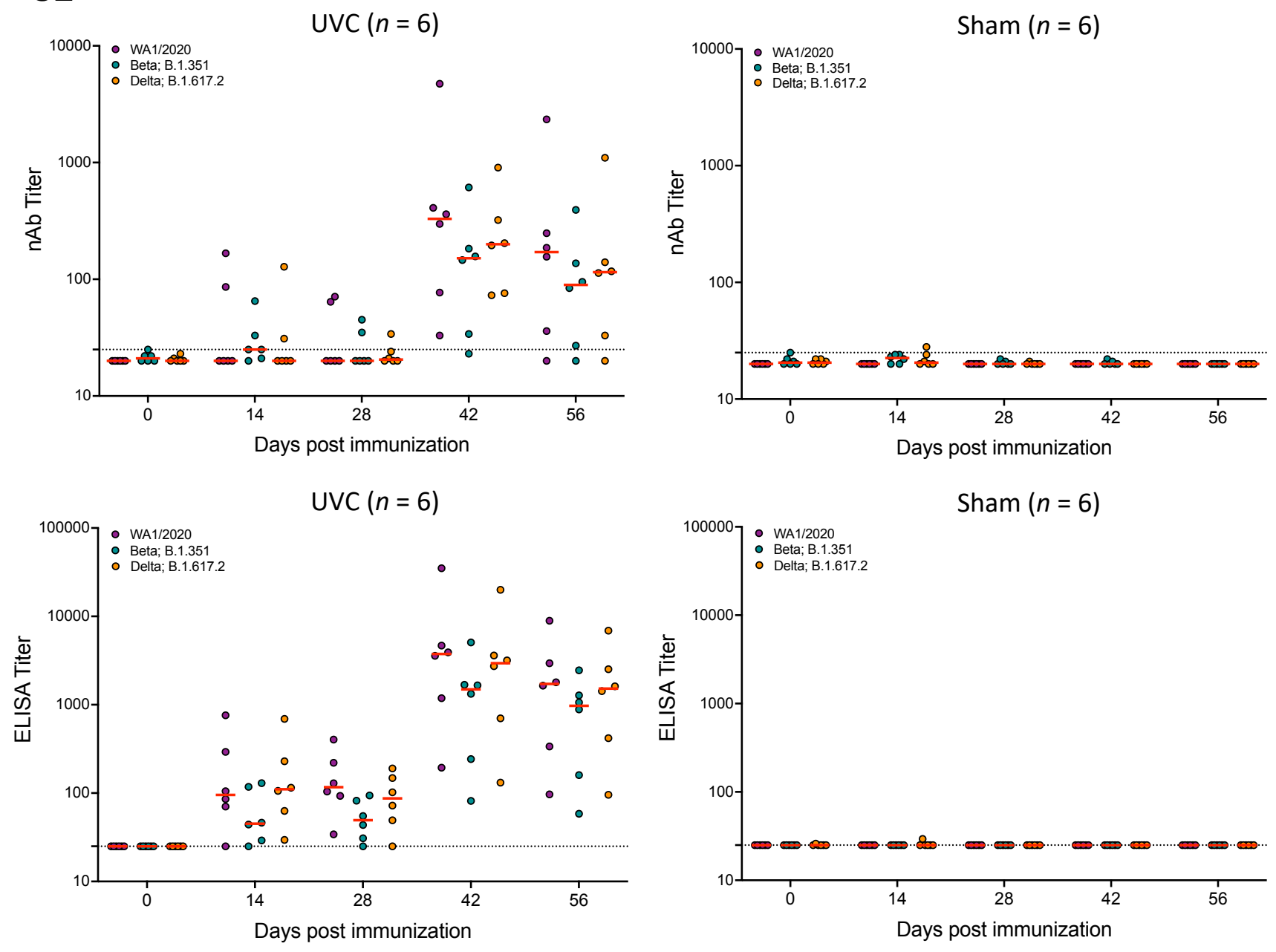